\title{
Establishing Consensus Turbulence Statistics for Hot Subsonic Jets
}

\author{
James Bridges ${ }^{*}$ and Mark P. Wernet ${ }^{\dagger}$ \\ NASA Glenn Research Center, Cleveland OH, 44135
}

\begin{abstract}
Many tasks in fluids engineering require knowledge of the turbulence in jets. There is a strong, although fragmented, literature base for low order statistics, such as jet spread and other meanvelocity field characteristics. Some sources, particularly for low speed cold jets, also provide turbulence intensities that are required for validating Reynolds-averaged Navier-Stokes (RANS) Computational Fluid Dynamics (CFD) codes. There are far fewer sources for jet spectra and for space-time correlations of turbulent velocity required for aeroacoustics applications, although there have been many singular publications with various unique statistics, such as Proper Orthogonal Decomposition, designed to uncover an underlying low-order dynamical description of turbulent jet flow. As the complexity of the statistic increases, the number of flows for which the data has been categorized and assembled decreases, making it difficult to systematically validate prediction codes that require high-level statistics over a broad range of jet flow conditions.

For several years, researchers at NASA have worked on developing and validating jet noise prediction codes. One such class of codes, loosely called CFD-based or statistical methods, uses RANS CFD to predict jet mean and turbulent intensities in velocity and temperature. These flow quantities serve as the input to the acoustic source models and flow-sound interaction calculations that yield predictions of far-field jet noise. To develop this capability, a catalog of turbulent jet flows has been created with statistics ranging from mean velocity to space-time correlations of Reynolds stresses. The present document aims to document this catalog and to assess the accuracies of the data, e.g. establish uncertainties for the data.

This paper covers the following five tasks:

- Document acquisition and processing procedures used to create the particle image velocimetry (PIV) datasets.

- Compare PIV data with hotwire and laser Doppler velocimetry (LDV) data published in the open literature.

- Compare different datasets acquired at roughly the same flow conditions to establish uncertainties.

- Create a 'consensus' dataset for a range of hot jet flows, including uncertainty bands.

- Analyze this consensus dataset for self-consistency and compare jet characteristics to those of the open literature.

One final objective fulfilled by this work was the demonstration of a universal scaling for the jet flow fields, at least within the region of interest to aeroacoustics. The potential core length and the spread rate of the half-velocity radius were used to collapse of the mean and turbulent velocity fields over the first 20 jet diameters in a highly satisfying manner.
\end{abstract}

\section{Nomenclature}

$\delta_{\omega} \quad$ shear layer thickness, $U_{j}|d U / d r|_{\max }$

$\eta \quad$ shear layer coordinate, $=\left(r-r_{0.5}\right) / \delta_{\omega}$

$\eta^{*} \quad$ shear layer growth rate coordinate, $=\left(r-r_{0.5}\right) / x$

$v \quad$ kinematic viscosity

$\theta \quad$ boundary layer momentum thickness at nozzle lip

$\psi \quad$ jet spread rate, $=\left(r_{0.5}-D_{j} / 2\right) / x$

$Y_{c} \quad$ jet spread factor, $=2 \psi x+D_{j}$

$c_{j} \quad$ speed of sound in jet fluid at jet exit, assuming fully expanded flow

\footnotetext{
${ }^{*}$ Acoustics Branch, MS 54-3, AIAA Associate Fellow

†Optical Instrumentation Branch, MS 77-1, AIAA Associate Fellow
} 


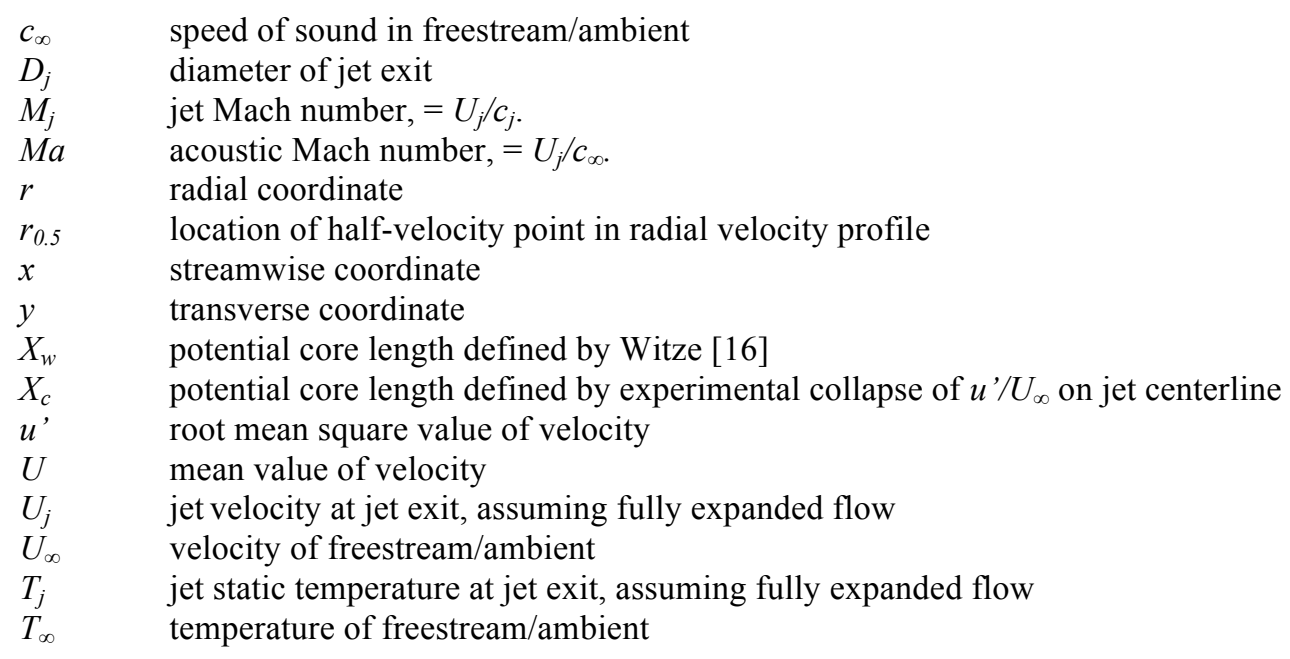

\section{Introduction}

Several articles in the open literature document previous efforts to measure turbulent jets. Most were acquired in cold, low speed jets, often using hotwire anemometry, although some results from laser Doppler velocimetry (LDV) were found and parts of these include measurements of hot jets. The data sources used are references 1 through 15 . Many of these were digitized and converted into a common format for comparison to the current PIV data.

The earliest source commonly cited for turbulence measurements in jets is Laurence in 1954 [1]. This detailed NACA report is detailed and deep, containing jet data of mean, variance, spectra, space and time correlations and length and timescale integrals for Mach numbers in a range from 0.2 through 0.7 . The data was acquired using the then-new technology of constant temperature anemometry and used variable length loops between tape heads to compute time-delay correlations. Unfortunately, when compared against the majority of data, it appears that the turbulence intensities were understated, perhaps as a result of uncorrected high frequency rolloff.

The early 1960's were an active time for jet noise-related turbulence measurements using hotwire anemometry, with Journal of Fluid Mechanics publishing extensive jet turbulence measurements by Davies, Fisher and Barrett in 1963 [2],Fisher and Davies [3, and Bradshaw, Ferriss and Johnson [4] in 1964. These papers contained an extensive host of data, from mean shear statistics to convective velocity and space-time correlations. Although the format of the Journal precluded extensive database reproduction and the depth also foreshortened how much data for any given statistic could be presented, these papers commonly presented radial and axial profiles of axial turbulence. Radial profiles were often presented for the shear layer, typically at $x / D_{j}=2$ or 4 which is well within the jet potential core, and normalized using $\eta^{*}=\left(r-r_{0.5}\right) / x$, where $r_{0.5}$ is the radial location where the jet mean velocity is half the profile peak value. Others have since chosen to normalize the radial profiles by local maximum shear gradient, or $\eta=\left(r-r_{0.5}\right) / \delta_{\omega}$, where $\delta_{\omega}$ is $U_{j} /|d U / d r|_{\max }$. The ratio between $\eta$ and $\eta^{*}$ is $x / \delta_{\omega}$ which is a measure of the shear layer spread and is roughly constant for two-dimensional shear layers. Davies et al., like Laurence, documented the impact of Mach number on basic turbulence levels. Given that the Mach numbers were below 0.5 and that the nozzles were 1" and 2" in diameter, it is possible that these measurements were subject to Reynolds number effects as well as the initial turbulence levels rather than compressibility effects. Bradshaw et al., working with cross-wires, documented all three components of turbulence. All three papers present extensive lengthscale estimates from correlation measurements.

In the early 1970's attention turned toward obtaining insight into what has become known as coherent structures in the jet flow. Papers such as Ko and Davies [5] explored the interesting inhomogeneities of the jet spectra, giving rise to interest in the structure of the jet as a way to simplify the description of the turbulence as it relates to jet noise. Use of external sources of perturbation to dominate the natural perturbations in the jet spawned several important studies. Crow and Champagne [6] in 1971 documented the impact of jet excitation on standard turbulence statistics, and introduced measurements that are useful in jet instability studies. In 1978 Raman, Zaman and Rice [7] produced useful basic turbulence statistics of jets as they explored the role of initial turbulence on the ability to excite a jet. Later in the 1970's not only were experimentalists turning their attention to new statistical descriptions to educe these large-scale structures, they were also using a new laser-based, non-invasive measurement technique: Laser Doppler Velocimetry (LDV). Of note here for the purpose of basic jet turbulence statistics are the papers of Lau and collaborators [8, 9, 10]. Lau, Morris and Fisher [8] compared the hotwire and LDV measurements and showed that at that time LDV measurements of turbulence were not quite comparable (figure 4 in the 
paper). However, this was soon rectified in subsequent papers and the advantage of the LDV at high speed and elevated temperature jets, allowed Lau [9] to document the impact of high speed on basic turbulence statistics, and Lau [10] extended the effort to hot jets. In this latter work Lau focuses mostly on gross features of spread rates and nondimensionalizing the jet potential core, but it is a data-dense paper even so. LDV also allowed an extensive dataset to be collected for unexcited and excited unheated and heated jets by Lepicovsky and collaborators, herein referenced through the NASA contractor report by Ahuja [11] in 1982.

With such extensive datasets available, few authors have documented the basic measures of jet turbulence in recent papers. One exception is Zaman [12], that documented the impact of lobed mixers on centerline statistics of the jet. Another exception is Kerherve et al. [13] that measured a supersonic jet in a forward flight environment. That work contains plots of radial turbulence intensity profiles at various axial stations in a shock-containing jet. It should be noted that they normalized the turbulence by the jet/ambient velocity difference $U_{j}-U_{\infty}$ and found a peak value of $17 \%$ at all stations. Most recently (2009), Morris and Zaman [14] have repeated measurements of turbulence in low speed jets with the objective of obtaining an extensive and consistent set of high order statistics, and in the process have documented their radial and centerline turbulence profiles.

Of course, while the new LDV technology brought about fresh measurements, so did the advent of PIV. Besides the current authors' papers, a notable work by Pokora [15] as carefully documented PIV measurements in a water jet, highlighting the differences found between two-and three-component PIV systems. Just as hotwire anemometry allowed measurement of unsteady velocity and LDV allowed measurement of high speed and hot jets, so did PIV bring about a multiorder of magnitude increase in information density. Instead of having roughly 10 points to define a profile now there are hundreds. Where once datasets could be written by hand in a single notebook, now it requires gigabytes of storage to hold the dataset of a single jet flow. And it has greatly enhanced the types of analysis that is possible. This has raised challenges in presenting the PIV datasets and making them available to interested researchers.

So just how do data from different sources compare? To find out, the graphs from the referenced papers were digitized and the data read electronically and a common dimensionless variable $u^{\prime} / U_{j}$ was plotted versus axial distance or radial coordinate. For axial distances we use both the jet diameter $\mathrm{D}_{\mathrm{j}}$ and the jet potential core length $X_{c}$ as normalization factors. The potential core length was computed using the formula of Witze [16]. The radial coordinate $r$, was normalized as local shear layer coordinate $\eta=\left(r-r_{0.5}\right) / \delta_{\omega}$ i.e., the radial coordinate has been normalized by the local mean velocity gradient as best as could be ascertained from the papers' data.

Turbulence is most commonly shown along the jet centerline, although this does not correspond to the peak turbulence level in the jet, another quantity of interest. In Figure 1, data from the literature covering a range of Mach numbers from 0.11 to 0.8 are plotted against axial distance normalized by jet diameter. This results in a rather large scatter among the data. In the figure, open symbols are hotwire data while the few closed symbols are LDV measurements. As the data are colored by Mach number, it is easy to see the effect of Mach number on where the peak occurs. When the axial coordinate is normalized by potential core length $X_{c}$ a satisfying collapse of the data results. A few of the outliers are surprising: The data of Bradshaw et al. and of Laurence are startlingly off given their citation count. Ignoring these two datasets, the peak axial turbulent velocity on the centerline is found to be 0.13 with a spread of 0.005 located roughly 1.5 potential core lengths downstream of the nozzle exit.

Because $x / D_{j}=4$ is a commonly used location for radial profiles, and because this location is not sensitive to variations in potential core length, data at this location were compiled for comparison of radial profiles for low subsonic cold jets, presented in Figure 2. Color is again used to separate the speeds, ranging from very small Mach for the water jet data of Pokora to $\mathrm{M}=0.5$. Filled symbols denote LDV measurements and an open $\mathrm{X}$ is the PIV data of Pokora, which falls outside the range of the other data. Ignoring this dataset, there is good agreement on the width of the shear layer, but a variation in peak amplitude of roughly $u^{\prime} / U_{j}=0.02$ out of 0.16 , or roughly $12 \%$ uncertainty. There is no improvement in this variation by concentrating on datasets containing only hotwire data sources. There is a bit of a systematic variation in peak amplitude with Mach number in the hotwire datasets. The LDV datasets, acquired at Mach numbers comparable to the highest of the hotwire measurements does not follow this trend. At this point it is possible that there is no valid trend with Mach number or that the LDV data is biased to higher turbulence amplitudes.

Radial profiles for cold jets of $\mathrm{M}$ greater than 0.5 are plotted in Figure 3. Data is presented from hot wire, LDV, and PIV velocimetry systems. Here the spread is roughly $50 \%$, with peak turbulence intensity ranging from 0.10 to 0.17 ! There is a clear bias with anemometry type, with the two LDV datasets being significantly above all the other data. There is no trend with Mach number or age of the data. At this stage it seems prudent to remove the excessively low hotwire data of Narayanan and perhaps weigh the LDV data a little less strongly than the preponderance of hotwire data that are in relative agreement. Doing so gives an estimate of peak axial turbulent velocity of 0.14 very near the half-velocity point, with a spread of roughly $-0.01 /+0.02$. 
Unfortunately, no more than one dataset of a hot subsonic jet could be located for either a centerline profile or a radial profile.
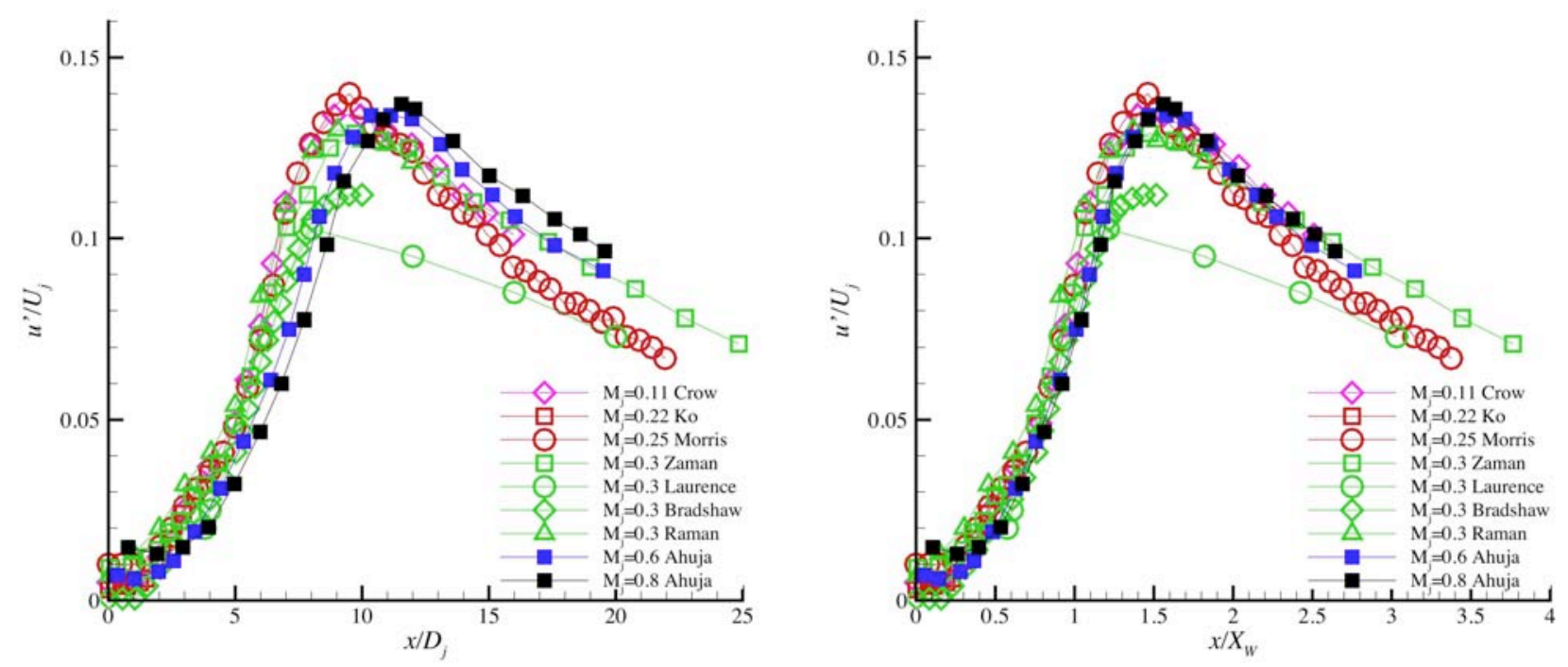

Figure 1 Axial profiles of axial turbulence on jet centerline as reported in the literature. Axial coordinate is normalized by jet diameter $D_{j}$ (left) and by potential core length $X_{W}$ (right).

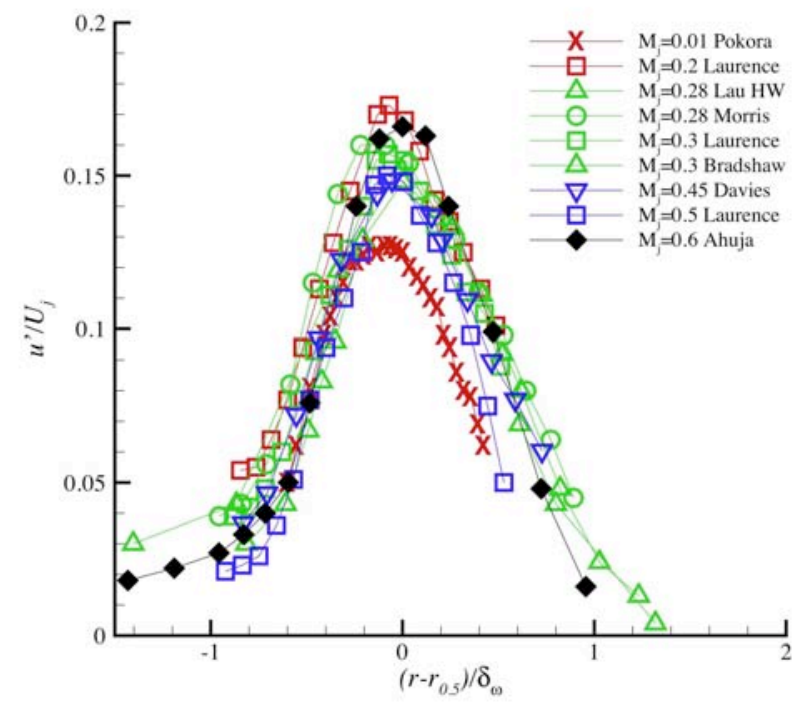

Figure 2 Radial profiles of axial turbulence at $x / D_{j} \sim 4$ for low subsonic, cold jets, as reported in the literature. 


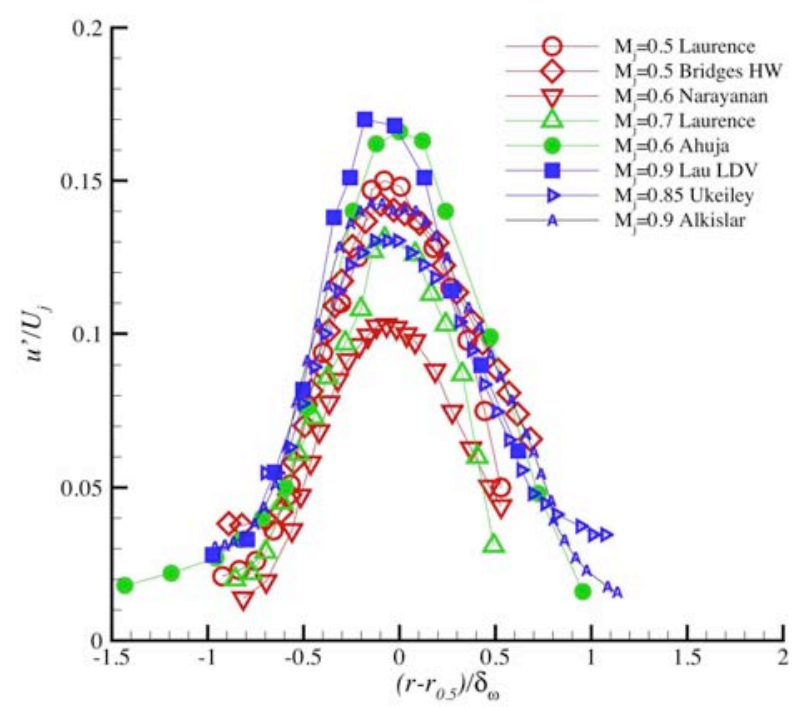

Figure 3 Radial profiles of axial turbulence at $x / D_{j} \sim 4$ for high subsonic, cold jets, as reported in the literature.

\section{Descriptions of NASA facility, PIV operations, test programs}

\section{A. Facility Description}

All original data presented in this document were acquired in the Small Hot Jet Acoustic Rig (SHJAR) at NASA Glenn Research Center. Other extensive datasets have also been acquired on a larger, higher fidelity jet rig, but for more complicated nozzle configurations that are harder to verify against independent sources. It is expected that, since the PIV and seeding systems are similar between the two rigs, uncertainties established by the present exercise can be carried over to the datasets acquired in the larger rig.

The Small Hot Jet Acoustic Rig (SHJAR, pronounced with a silent ' $J$ '), shown in Figure 4, is located in the Aeroacoustic Propulsion Laboratory (AAPL) at the NASA Glenn Research Center in Cleveland, Ohio. The SHJAR was developed to test jet noise reduction concepts at a low technology readiness level (TRL 1-3) and at minimum expense. The AAPL, which houses the SHJAR, is a geodesic dome (60-foot radius) lined with sound absorbing wedges which reduce sound reflection at all frequencies above $200 \mathrm{~Hz}$. The jet exhaust is directed outside through a large door that permits unrestricted flow seeding operation without introducing noise interference concerns related to particulate collection systems.

As a jet noise testing rig, the SHJAR was designed to minimize rig noise sources, incorporating the recommendations of Viswanathan [17] and Ahuja [18] to achieve this goal. The rig is a single flow jet rig that used 150-psi air supplied by several remotely located compressors. The maximum mass flow rate was $6 \mathrm{lbm} / \mathrm{second}$ and the maximum temperature air was 1300 ${ }^{\circ} \mathrm{F}$. A hydrogen-fueled combustor permits a large range of temperature flows to be tested. The air passes through a baffled muffler and settling chamber before it reaches the nozzle. Two valves, a large main valve and a small vernier valve located upstream of the combustor and muffler, control the rate of airflow, providing fine control over the entire range of operating conditions. Flow conditions are measured in the 14" $(356 \mathrm{~mm})$ diameter plenum chamber located within the supporting frame. An ASME contraction connects the plenum chamber to a 6" $(150 \mathrm{~mm})$ diameter nozzle feedpipe that is 25 " $(635 \mathrm{~mm})$ long. An extensive study of the acoustic properties of the SHJAR rig and its validation are given in Brown and Bridges [19]. 


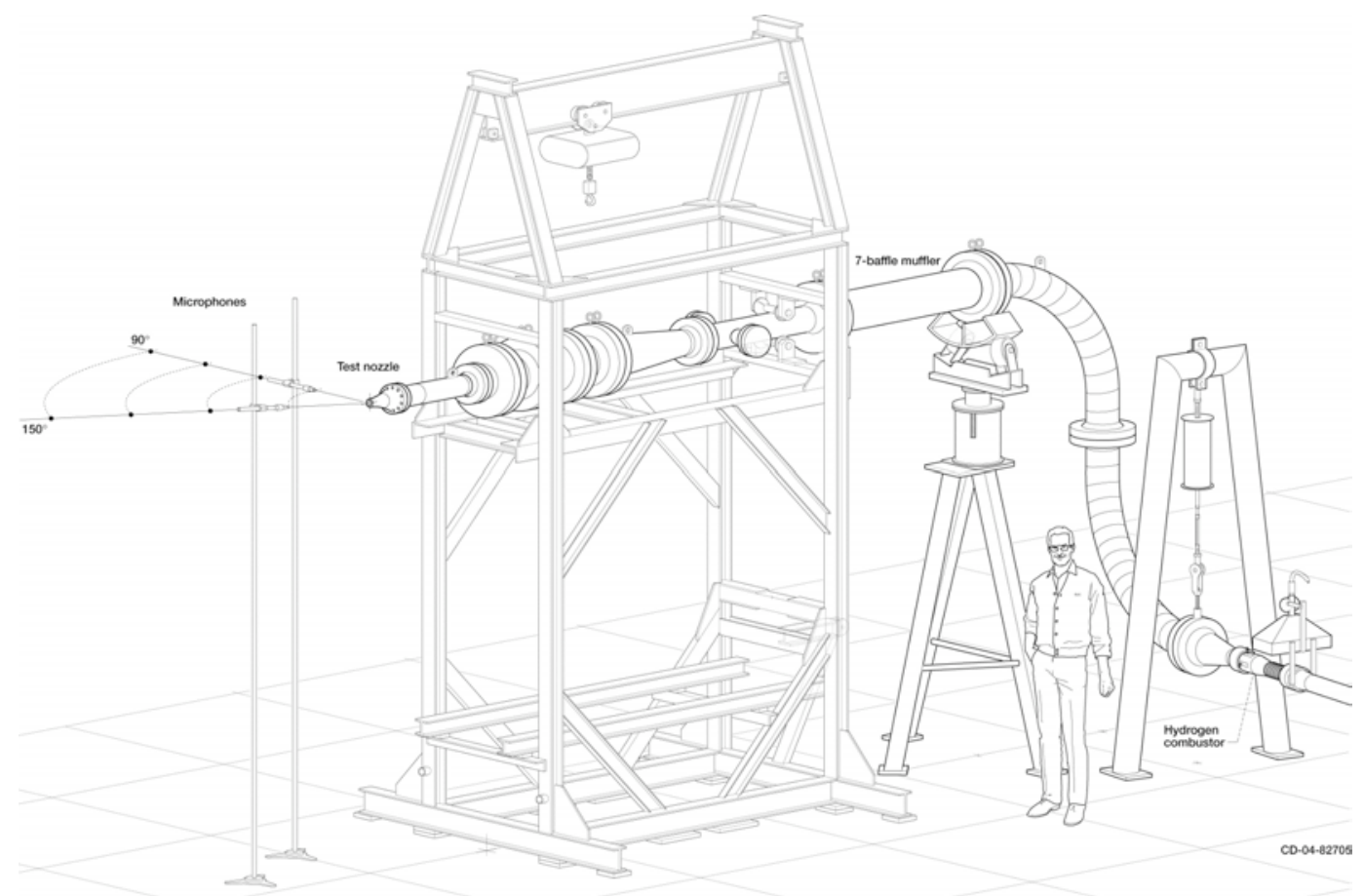

Figure 4 Small Hot Jet Acoustic Rig (SHJAR).

\section{B. Test Nomenclature}

This report covers testing done from October 2001 through August 2007. Roughly once a year a test campaign was mounted which required PIV testing in the SHJAR. With each entry the implementation of PIV was changed in some way, to either address lessons learned from previous testing or to add new capability, such as a third velocity component, faster data acquisition rate or $\mathrm{kHz}$ time resolution. Whenever possible, data for a matrix of points was acquired, aiming to duplicate, or improve upon, previous measurements. This repeat of conditions with varying PIV configurations has allowed a certain insight into data uncertainty that is very valuable for engineering practice.

The set of flow conditions that were chosen for repeat testing was a subset of the matrix created by Plumblee et al [20] (conventionally known as the Tanna matrix). This matrix is defined on constant acoustic Mach number Ma (jet velocity normalized by ambient speed of sound) and constant static temperature ratio $T_{j} / T_{\infty}$ (static temperature relative to ambient temperature). The matrix of flow conditions considered in the current report is given in Table 1. Table 2 lists the test entries, which were known by mnemonics that usually include the NASA fiscal year and some indication of the test content. For this report the test entries have been labeled T0 through T6 and will be referred to in describing datasets, experimental setups, etc. Under the heading 'PIV Configuration', the table notes whether the data plane is streamwise $(X, Y, Z=0)$ or cross-stream $(\mathrm{X}=\mathrm{c}, \mathrm{Y}, \mathrm{Z})$, whether two or three components of velocity were acquired, and whether there was temporal measurements available, either as fixed time delays ( $\mathrm{T}=\mathrm{tau}$ ) or for a continuous record in time $(\mathrm{T})$. The table also lists the nozzles tested and some insight into the original purpose of the test.

Table 1 Definition of test conditions.

\begin{tabular}{|c|c|c|c|c|}
\hline Set point & Ma & $\mathbf{T}_{\mathbf{j}} / \mathbf{T}_{\infty}$ & $\mathbf{N P R}$ & $\mathbf{M}$ \\
\hline $\mathbf{3}$ & 0.500 & 0.950 & 1.197 & 0.513 \\
\hline $\mathbf{7}$ & 0.900 & 0.835 & 1.861 & 0.985 \\
\hline $\mathbf{2 3}$ & 0.500 & 1.764 & 1.102 & 0.376 \\
\hline $\mathbf{2 7}$ & 0.900 & 1.764 & 1.357 & 0.678 \\
\hline $\mathbf{2 9}$ & 1.330 & 1.764 & 1.888 & 1.001 \\
\hline $\mathbf{4 6}$ & 0.900 & 2.700 & 1.219 & 0.548 \\
\hline $\mathbf{4 9}$ & 1.485 & 2.700 & 1.678 & 0.904 \\
\hline
\end{tabular}

Table 2 Description of test entries 


\begin{tabular}{|c|c|c|c|c|c|}
\hline $\begin{array}{l}\text { Test } \\
\text { Entry }\end{array}$ & Test Name & Dates & PIV Configuration & $\begin{array}{l}\text { Nozzles } \\
\text { tested }\end{array}$ & Purpose \\
\hline T0 & SHJARCHK & Oct 2001 & $\begin{array}{l}X, Y, Z=0 \\
U, Y\end{array}$ & $\begin{array}{l}\text { ARN1, } \\
\text { ARN2 }\end{array}$ & Initial checkout of SHJAR \\
\hline T1 & SHJARBASE1 & Apr 2002 & $\begin{array}{l}\mathrm{X}, \mathrm{Y}, \mathrm{Z}=0, \mathrm{~T}=\mathrm{tau} \\
\mathrm{U}, \mathrm{V}\end{array}$ & ARN2 & $\begin{array}{l}\text { Dual-PIV for space-time } \\
\text { correlations }\end{array}$ \\
\hline T2 & SHJARCHEV04 & Nov 2003 & $\begin{array}{l}\mathrm{X}=\mathrm{c}, \mathrm{Y}, \mathrm{Z} \\
\mathrm{U}, \mathrm{V}, \mathrm{W}\end{array}$ & $\begin{array}{l}\text { ARN2, } \\
\text { SMC00x }\end{array}$ & $\begin{array}{l}\text { Cross-stream, three- } \\
\text { component }\end{array}$ \\
\hline T3 & SHJARBASE2 & Dec 2003 & $\begin{array}{l}X, Y, Z=0 \\
U, V, W\end{array}$ & $\begin{array}{l}\text { ARN2, } \\
\text { SMC00x }\end{array}$ & $\begin{array}{l}\text { Streamwise, three- } \\
\text { component }\end{array}$ \\
\hline T4 & SHJARBASE05 & Jan 2004 & $\begin{array}{l}\mathrm{X}, \mathrm{Y}, \mathrm{Z}=0 \\
\mathrm{U}, \mathrm{V}, \mathrm{W}\end{array}$ & $\begin{array}{l}\text { SMC000, } \\
\text { SMC01x }\end{array}$ & $\begin{array}{l}\text { Streamwise, three- } \\
\text { component, supersonic } \\
\text { nozzles }\end{array}$ \\
\hline T5 & TRPIV06 & May 2006 & $\begin{array}{l}\mathrm{X}, \mathrm{Y}, \mathrm{Z}=0, \mathrm{~T} \\
\mathrm{U}, \mathrm{V}\end{array}$ & SMC000 & Time-resolved PIV \\
\hline T6 & TRPIV07 & $\begin{array}{l}\text { Jun-Aug } \\
2007\end{array}$ & $\begin{array}{l}\mathrm{X}, \mathrm{Y}, \mathrm{Z}=0, \mathrm{~T} \\
\mathrm{U}, \mathrm{V}\end{array}$ & $\begin{array}{l}\text { SMC000, } \\
\text { SMC01x }\end{array}$ & $\begin{array}{l}\text { Time-resolved PIV on } \\
\text { supersonic nozzles }\end{array}$ \\
\hline
\end{tabular}

\section{Nozzle Description}

Some of the nozzles used in this report were from a family of convergent nozzles, called the Acoustic Reference Nozzles (ARN), designed to be simple to characterize with similar dimensions such as inlet diameter $(6.0 " / 15.24 \mathrm{~mm})$, lip thickness $(0.050 \% / 1.27 \mathrm{~mm})$, outside face angle $\left(30^{\circ}\right.$ to jet axis), and parallel flow section at the exit $(0.25 \% / 6.4 \mathrm{~mm})$. In some data reported here, acquired before 2003, the ARN1, a 1"/25.4mm diameter nozzle, and ARN2, a 2"/50.1mm diameter nozzle, were used. These are shown connected to the feedpipe in Figure 5 .

The ARN series of nozzles featured a relatively strong contraction that resulted in relaminarization of the boundary layer, especially at low Reynolds numbers, and potentially caused some Reynolds number dependence of the jet's behavior. Since the point of nozzle testing is to simulate large-scale nozzles, e.g. high Reynolds number, with initially turbulent shear layers, it is desired to avoid relaminarization in the nozzle contraction.

To avoid relaminarization and to provide a baseline for a series of simple chevron nozzles, the Small Metal Chevron (SMC) nozzle system was developed. Originally conceived as a model system for parametric testing of chevron nozzles, its modular design has lent itself to a large number of nozzle concepts being mounted on it, including chevrons and convergentdivergent nozzles. The baseline axisymmetric convergent nozzle, SMC000, is shown in Figure 6. It has an exit diameter of $2 " / 50.8 \mathrm{~mm}$.
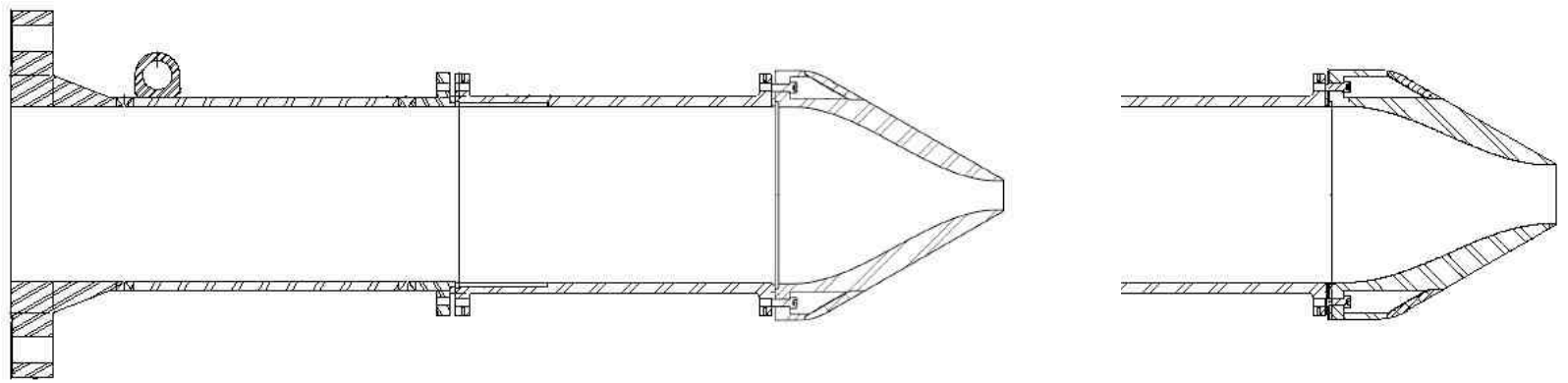

Figure 5 SHJAR nozzle system with Acoustic Research Nozzle 1 (ARN1) and 2 (ARN2) attached.

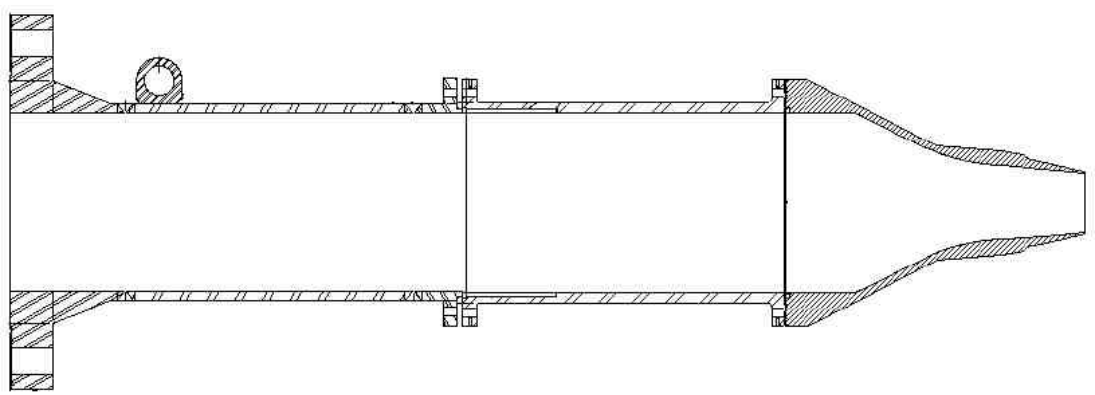

Figure 6 SHJAR nozzle system with SMC000 nozzle attached. 
In 2004 and 2005, a series of measurements were made with hotwire anemometry to characterize the exit boundary layer/initial shear layer of the ARN and SMC nozzles by measuring velocity profiles at the nozzle exits at low Mach numbers. Classically, one expects that laminar boundary layers will have a shape which, when quantified by the ratio of momentum thickness and displacement thickness, will produce a value, called the shape factor, of roughly 2.5. Fully turbulent boundary layers have shape factors closer to 1.8. Another indicator of an initially turbulent shear layer is the turbulence intensity, $u^{\prime} / U_{j}$, which ranges from immeasurably small for a truly laminar case, to as much as $10 \%$ for an intensely turbulent boundary layer.

Figure 7 contains three plots from these hot wire tests, one each with the shape factor, the peak turbulence intensity, and the momentum thickness as a function of Reynolds number, $R e_{D}=U_{j} D_{j} / v$. Two nozzles are shown, although different upstream treatment has been applied to these nozzles, as specified in the plot legends. The ARN2 and SMC000 nozzles were measured in both 2004 and 2005, but between the years (in April 2004, specifically; between test entries T3 and T4) a series of screens 12"-14" upstream of the nozzle were removed. In both cases this slightly raised the shape factor (more laminar). It also strongly decreased the peak turbulence. This was not appreciated at the time since other factors, including acoustics, showed that the smc000 was not suffering from known laminar artifacts. For comparison, the ARN2 nozzle was tested with a significant trip placed in the contraction and the boundary layer parameters for this very turbulent initial condition are shown in the plots. This configuration was not tested using PIV. The impact of the screen removal on the flow can be determined by comparing to SMC000 data acquired in the T3 and T4 test programs. In section V.B below it will be concluded that no difference could be determined from looking at plots of mean velocity or turbulence intensity.
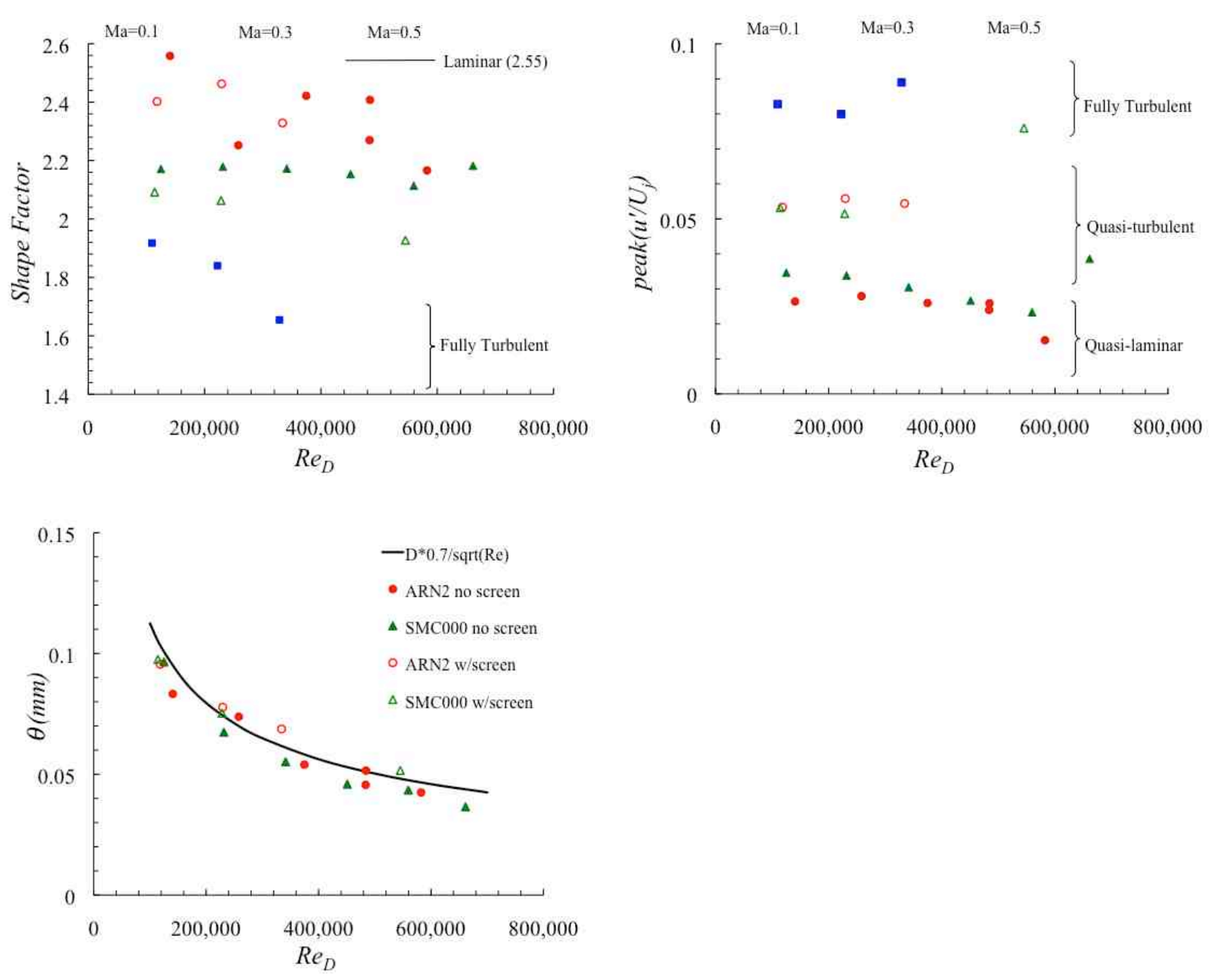

Figure 7 Boundary layer statistics (shape factor, peak turbulence level, momentum thickness) for ARN2 and SMC000 nozzles for low Reynolds numbers $(0.1<M a<0.5)$. 


\section{Particle Image Velocimetry Systems}

Details of the various PIV installations are given in references 21, 22, 23. Below are descriptions of aspects of the PIV installations, designed to show what aspects were in common and what aspects changed between tests.

\section{Two-component PIV}

In the initial tests (T0, T1) a two-component PIV systems with the light sheet oriented parallel to the flow direction were used. In the T0 entry the PIV system components were located on the traversing mechanism, colloquially known as Big Blue, shown in Figure 8. Nozzle flow surveys were obtained by traversing the PIV system along the jet axis using the large traverse system. A Continuum PIV-Surelite III, $400 \mathrm{~mJ}$ per pulse at $532 \mathrm{~nm}$, dual head, Nd:YAG laser operating at $10 \mathrm{~Hz}$ produced the pulsed light sheets. A laser light sheet approximately $0.2 \mathrm{~mm}$ thick was formed with one cylindrical and one spherical lens and directed along the jet axis. A Redlake ES 4.0 digital camera with a $2048 \times 2048$ pixel CCD array was used to record the PIV images. The PIV camera was configured for a $136 \mathrm{~mm}$ field. Particle displacements ranging from 0 to 17 pixels were measured, resulting in a full-scale error of $0.5 \%$ after subpixel particle image fitting. Four hundred image pairs or velocity maps were acquired for each axial location and were used in the statistical processing of the turbulence data. The full-scale errors given above were, therefore, reduced further through the averaging process. The PIV images were processed with PIVPROC (reference 25), a NASA GRC-developed code. PIVPROC uses a correlation-based processing algorithm that allows for subregion image shifting and multi-pass correlation to improve the spatial resolution of the resultant velocity vector maps. The first pass of the correlation used a subregion size of $64 \times 64$ pixels with $50 \%$ overlap. The second pass used a $32 \times 32$ pixel subregion size, again with $50 \%$ overlap. The resulting spatial resolution of the velocity vector fields was $1.06 \mathrm{~mm}$ for the $136 \mathrm{~mm}$ fields of view. The complete stereo PIV system, including all cameras and backdrops, data acquisition computers, laser hardware and optics were rigidly mounted on a large axial traverse located downstream of the nozzle exit plane. The travel range of the traverse was approximately $2.5 \mathrm{~m}$, with a positioning accuracy of $1.0 \mathrm{~mm}$.
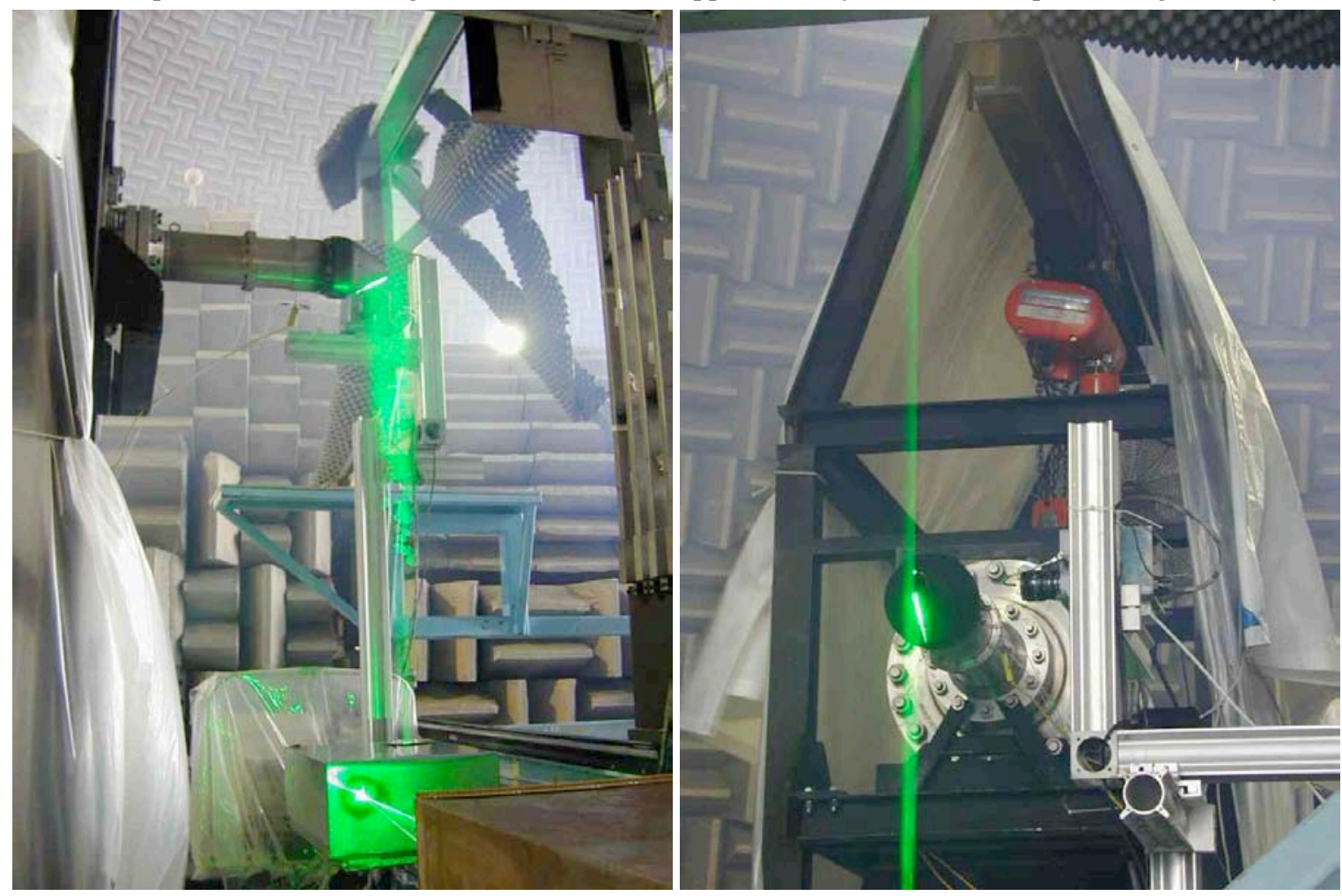

Figure 8 PIV setup for test entry T0. Left: Laser and beam-forming optics below, light sheet impinging upon bottom of nozzle, black velvet covered optical background for cameras in upper left of image. Right: Light sheet impinging upon nozzle, camera mounted 12" away, 'tent' around the SHJAR frame to guide ambient seeding from oil vapor smoke generator. Eggshell foam on the rig is for acoustic measurements - not PIV.

\section{Stereo PIV Optical Calibration and Verification}

For test entries T3 and T4 a stereo PIV installation was deployed. Each camera was oriented at a certain angle with respect to the laser sheet to obtain a stereo view of the measurement plane. Because of the incorporation of the Scheimpflug 
condition, magnification factors between the image and object planes are variable due to perspective distortion. In order to combine camera measurements from both views into a single, three-component velocity vector map in the fluid plane, camera calibrations were required. The three-dimensional in-situ calibration procedure as outlined by Soloff et al. [24] was performed.

Velocity vector maps for each camera were computed in the image planes from the image pairs using NASA developed PIVPROC software [25]. The software utilizes conventional multi-pass PIV cross-correlation processing algorithms and incorporates error detection based on image correlation signal to noise ratio. First pass interrogation region sizes of 64 x 64 pixels on 32 pixel centers and final pass interrogation region sizes of $32 \times 32$ pixels on 16 pixel centers were used to process image pairs from the cameras in both stereo configurations.

After processing the images acquired by each camera, the pixel displacement data from the left and right views were combined to obtain the three-component velocity vector field at the measurement plane in the fluid. The equations that govern the transformation of the image plane data to the object plane result from the verified calibration procedures noted above. The software used to perform these operations was developed in-house to permit execution via command line processing. For the large volumes of data typically acquired during these measurements, batch mode processing is quite useful and and makes efficient use of all available CPUs on the computer. Some notable features include the ability to perform bicubic vector interpolation of the neighboring vector data in the image plane prior to being mapped back into the fluid space, as well as the ability to calculate three-dimensional vectors in the fluid on arbitrarily defined rectangular grids of any size - useful for non-square correlation region processing.

As the u-, v-, and w-components of each three-dimensional (3D) velocity vector are computed using a linear least-squares fit (singular value decomposition, or SVD) of the left and right 2D vector, a residual error is found by substituting each solution back into the least-squares equations. The total residual error for each vector is given as the square root of the sum of these residual errors squared. This error, given in pixels, is indicative of the total mismatch between left and right vectors. A residual pixel threshold of 0.5 pixels was specified for validating the solution vector. For the cross-flow configuration, solutions exceeding this threshold indicate the cross-stream components of velocity between the two vectors differed by more than 0.5 pixel.

Ensemble averaging of the 200 individual vector maps was performed to obtain statistical information at each measurement plane. The averaging procedure incorporated both hard velocity cut-off limits and Chauvenet's criterion for data outlier removal [25]. This ensemble averaging was also performed using in-house developed software, with any 3D vector data exceeding the residual pixel threshold ( 0.5 pixels) being excluded from the ensemble.

\section{Temporally-Resolved PIV}

By test entry T5 a new possibility in PIV had appeared: high speed, time-resolved PIV (TR-PIV). The first attempt, test T5, required significant changes to the PIV setup. First, to achieve the high rates of acquisition, a powerful, high pulse rate laser was substituted. The power supplies alone increased weight on the traverse from $\sim 40$ to $200 \mathrm{lbs}$. More significantly yet, the laser sheet, whose spread had to be minimized to maintain sufficient energy, was projected from directly downstream of the jet. This left the optics being blasted by the seeded hot jet! A sheet metal box with sacrificial window around the optics, purged with high pressure nitrogen, kept the optics clean for long enough to acquire the few minutes data for each flow.

The TR-PIV system implementation for this nozzle shear layer test represents an optimization of the available laser pulse energy and the flexibility afforded by the CMOS cameras to select a high aspect ratio region-of-interest at high framing rates. To measure a long spatial extent of the nozzle shear layer flow, the TR-PIV system was configured so that the laser light sheet propagated along the jet axis. By using a pair of CMOS cameras mounted side-by-side at the nozzle lip line height, the axial extent of the flow field being imaged was maximized. Forming the pulsed laser beam into a short height, thin laser light sheet ensured that there was sufficient energy density across the CMOS cameras' field of view so that the light scattered by the submicron sized seed particles in the flow could be detected.

The CMOS cameras used in this study were Photron Ultima APX-RS cameras, which have $17.5 \mu \mathrm{m}$ square pixels in a 1024 by 1024 pixel sensor. Each camera is capable of operating at $3000 \mathrm{fps}$ at full resolution. Higher framing rates are available at reduced resolution. The primary objective of this test was to obtain time resolved PIV measurements of the flow at $10 \mathrm{kHz}$, with a secondary objective of $25 \mathrm{kHz}$ measurements. For this report, only data acquired at $10 \mathrm{kHz}$ are reported. In order to acquire 'frame-straddle' image pairs at $10 \mathrm{kHz}$, the Photron cameras were operated at a framing rate of $20 \mathrm{kHz}$. In the remainder of the text, any reference to $10 \mathrm{kHz}$ PIV implies that the cameras were operated at a frame rate of $20 \mathrm{kHz}$ with the laser pulses synchronized to 'frame straddle' the image frame pairs at $10 \mathrm{kHz}$. The combined dual camera configuration had a 144 by 2048 pixel field of view at $20 \mathrm{kHz}$. The cameras were equipped with $85 \mathrm{~mm}$ focal length lenses with $1.4 \mathrm{X}$ teleconverters to obtain the desired $19 \mathrm{~mm}$ by $150 \mathrm{~mm}$ field of view from each camera at $20 \mathrm{kHz}$. The cameras were each equipped with 8 gigabytes of on board memory and a gigabit Ethernet interface for downloading the acquired image data. Although the image acquisition time was slightly over $2.2 \mathrm{~s}$, the image download time was typically $15-19 \mathrm{~min} / \mathrm{camera}$. 
The pulsed light sheet illumination was provided by a Quantronix Infini dual head laser system. The laser heads and beam combining optics are housed in a single enclosure. The Infini laser is lamp pumped and uses an RF driven Q-Switch to generate the $10 \mathrm{kHz}$ pulses from each laser head. The nominal output pulse energy is $6 \mathrm{~mJ} / \mathrm{pulse} / \mathrm{head}$ at $10 \mathrm{kHz}$. The beam has a beam quality $\mathrm{M}^{2}<16$ and the pulse length is $130 \mathrm{~ns}$ at full width half maximum. The laser beam propagated downstream, parallel to the jet axis, to the light sheet forming optics, as shown in Figure 9. Pairs of cylindrical and spherical lenses were required in order to form the laser beam into a 20 by $2 \mathrm{~mm}$ light sheet at the measurement plane.

The test matrix required measurements in both the shear layer and along the nozzle centerline in the potential core of the jet. Measuring the shear layer flow along the nozzle lip line necessitated propagating the laser light sheet from a downstream location back upstream to the nozzle exit. The light sheet was vertically centered on the nozzle lip line. The laser head was mounted so the beam propagated in a downstream direction. A $100 \times 100 \mathrm{~mm}$ box beam was mounted on the large traverse to support the light sheet forming optics. The laser beam was turned $90^{\circ}$ along the box beam, where the light sheet forming optics were mounted. The light sheet optics were enclosed in a sheet metal housing to protect them from the hot, seeded jet exhaust. A fused silica window was placed at the exit of the enclosure. A nitrogen purge stream of air was directed over the face of the exit window in order to keep it clear of seed material. In addition to the nitrogen purge, a right angle sheet metal wedge was fabricated to protect the exit window (see Figure 9). The protective wedge had a slot cut out for the exiting laser beam and was flooded with $120 \mathrm{psi}$ air to prevent seed material from entering the enclosure and coating the exit window. The laser beam propagated $2.1 \mathrm{~m}$ upstream from the sheet forming optics to the nozzle. Figure 9 shows the layout of the TR-PIV system and a photo of the system in operation.

Processing of the TR-PIV data was performed using the latest innovations in PIV data reduction. A simulated annealing algorithm is used in conjunction with Subregion Distortion processing. The sub-region image shifting results in the correlation peak being nearly centered on the correlation plane. In the simulated annealing process, the region on the correlation plane that is searched for the correlation peak is successively reduced to remove random noise effects. The simulated annealing processing is then followed by subregion distortion processing, where the local velocity field is used to distort the correlation subregions before performing the cross-correlation computation. As a result the TR-PIV data are some of the highest quality PIV data processed to date.

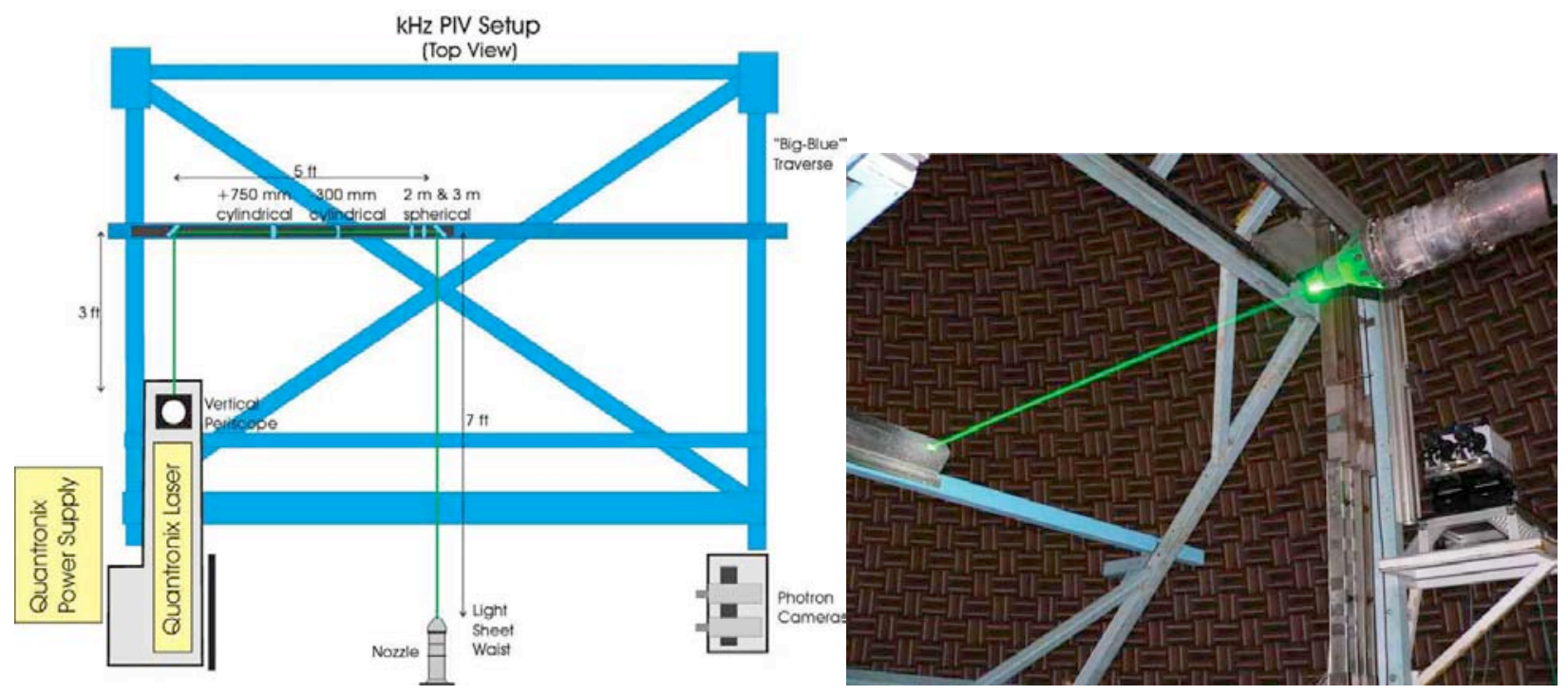

Figure 9 TRPIV setup for T5 and T6 entries. Left: schematic of optical layout. Right: picture of the setup in the SHJAR facility showing the cover over the light sheet forming optics, the light sheet impinging on the nozzle lip and the two Photron cameras.

\section{E. Seeding}

As is typical in all PIV applications, quality flow seeding was an essential factor in obtaining high accuracy results. Due to the elevated operating temperatures, a refractory seed material was required for the core jet flow. The flow seeding material utilized in all test entries was $\sim 0.5$ micron alumina particles [26]. This material, carried within a pH adjusted ethanol solution, was delivered to the core flow upstream of the flow conditioning screens. Uniform dispersion was provided by a pair of air-assisted, atomizing nozzles. As determined from a particle frequency response analysis, the core flow particles were expected to be able to accurately follow the jet flows over the complete range of nozzle operating conditions considered 
in these test programs. Uniform seeding in a jet flow is essential and difficult. This was especially important when using polarization in test $\mathrm{T} 1$ as the size of the particles must be small enough to follow the flow and to maintain the polarization of the incident light upon scattering.

The ambient air was seeded with $0.2-0.3$ micron mineral oil droplets produced by a commercial smoke generator. This ambient smoke system was located in a partially enclosed rig support structure upstream of the nozzle as seen in Figure 8 . Not shown is a variety of simple fans used to adjust the rate at which the seeded air was forced out of the tent to engulf the jet ambient. This provided a very low velocity $(<5 \mathrm{~m} / \mathrm{s})$ air stream surrounding the nozzle core jet flows. After several iterations on ambient seeding arrangements, the method of releasing oil droplet 'smoke' from a commercial fogger that essentially replicated a very low velocity freejet around the research jet was optimized. With this set of seeders we achieved seeding adequate for good velocity vector determination at each point in the map over $99 \%$ of the time.

Finally it should be noted that given the optical setup and the particle size the cameras were not actually imaging the particles. This is advantageous because the particles images would have been less than a pixel in size, leading to a problem with peak locking in the image correlation processing. The effect of 'defocusing' the image system was to allow the particle image to cover more than a pixel, assuring the correlation algorithm of finding a parabolic peak in the correlation domain from which a subpixel peak could be determined.

\section{F. Note About Data Quality}

In PIV work described here, time averages are computed as ensemble averages over uncorrelated instantaneous captures of velocity fields. In processing the images to velocity maps several criteria are used to determine if an instantaneous velocity vector is valid and to remove those vectors that are 'obviously bad'. Generally speaking, the more points have to be thrown out, the more suspect the data is. An important statistic for diagnostic purposes is the data quality, defined as the number of accepted vectors at a point relative to the total number of vector maps acquired. For the data used in computing the single and two-point statistics presented here there were only a few regions where the quality was below 0.95 . Most of these occur near edges of the individual images that make up the composite. Statistics in these regions may be corrupted by bad points, making the data less significant in subsequent analysis. In computing composite maps, statistics from the various overlapping regions were combined using data quality weighting to reduce the error in the composite statistic.

Another issue that can plague PIV measurements is peak locking, a phenomena where subpixel determination of correlation peaks is defeated by having images where the particle images do not cover more than a single pixel on the camera. This is a very real problem with large fields of view such as are needed in computing spatial correlations. This problem can be checked by looking for peaks corresponding to integer pixel displacements in the histogram of velocities over an image and slightly defocusing the image during acquisition if needed.

In test program T0, 400 velocity fields were available for statistical analysis at each location. For test program T1 a compromise was made for having two PIV systems and only 200 fields were available. In entries T2-T4, 200 fields were used. In T5 and T6, roughly 22,000 fields were acquired for the configurations presented here. At the $10 \mathrm{kHz}$ sample rate, however, these are not independent samples, but still represent many hundreds of independent samples relative to the dominant timescales of the jets.

When trying to establish uncertainty bands for the statistics computed from the PIV data, several factors were taken into consideration and grouped into bias and random errors. Bias errors include optical calibration of the PIV setup, subtle issues with seeding size and density, image analysis procedures, rig flow instrumentation, and unique aspects of the particular nozzles used in the test. With a large number of different PIV configurations and analysis procedures used over many years' time, much of the potential for bias error has been removed from this data. The comparison with statistics obtained by other anemometry techniques, as reported in open literature is the main method used in this report to measure the bias error that might remain. Contributing to the random error were the uncertainties in the instantaneous measurements, the uncertainties associated with the number of ensembles used, and the uncertainties in rig instrumentation as outlined above. These errors are easier to address because they can be quantified using repeatability and reduced by increasing the amount of data in the ensembles. To reduce random error spatial averaging was performed in the streamwise direction, which takes advantage of the high spatial density of the PIV measurements compared with the relatively slowly varying statistics in the axial direction of the jet. Another tactic, which addresses both bias and random error was the decision to average the two sides of the jet on the assumption of symmetry. Variations from one test entry to another, along with internal image processing diagnostics ( the 'quality' metric) were used to measure this error for the data in this report.

\section{Comparisons of NASA PIV data with literature}

Datasets from seven PIV test entries are considered here. Greatest focus is on tests T0, T1, T3, T4 that used streamwise light sheets. There was sufficient repeats of subsonic Tanna cases to evaluate repeatability, and several of these tests 
measured setpoint 3, the Mach 0.5 cold jet case, for comparison to hotwire data in the literature. There were also many repeats of setpoint 7 , the $M a=0.9$ cold jet case $\left(M_{j}=0.98\right)$, which can be compared to high subsonic unheated jets. Finally, both Lepicovsky and Lau measured high subsonic jets heated to static temperature ratios near 2.5, a close match to setpoint 46 that was measured in two of the test entries.

Setpoint 3 data from all the different PIV test programs were extracted along the centerline and along a radial profile at $\mathrm{x} / \mathrm{D}=4$ to compare with the subsonic, cold jet data from the literature. As shown in Figure 10, the PIV datasets fall within the data scatter from the hotwire and LDV data, a band of roughly $u{ }^{\prime} / U_{j}= \pm 0.01$ near the peak. In the radial profiles, the PIV data fall well within the data of the literature, showing no bias.

At setpoint 7 (Figure 11), the scatter in the PIV datasets is much greater, partly because data from the two TR-PIV runs, T5 and T6, have been added. These have smoother profiles but tend to be lower than the conventional PIV data. Not counting these two entries, the PIV data are centered on the literature data in the axial profile. The radial profiles are much cleaner with the later test entry data falling 0.015 lower than the LDV data at the peak. Although there is some concern about the accuracy of the early LDV data in the literature, it appears possible that the PIV data may be as much as $10 \%$ low in the peak turbulence region of the high subsonic cold jet.

There is very little data for hot jets, most of which come from measurements in the early 80's. The data of Lepicovsky in Ahuja's report [11], and of Lau's 1981 JFM paper [10] was used to assess the bias of jet turbulence values for axial and radial profiles, respectively. Lepicovsky documented centerline velocity statistics for a $M j=0.78\left(U_{j}=428 \mathrm{~m} / \mathrm{s}\right), 800 \mathrm{~K}$ jet, but no radial profiles. Lau 1980 gives radial profiles for a $M_{j}=0.6, \mathrm{~T}_{\mathrm{j}} / \mathrm{T}_{\infty}=2.32$ jet, but no axial profiles. In the plots of Figure 12 , the axial turbulence $u^{\prime} / U_{j}$ of the PIV datasets are seen to be within 0.01 of the LDV datasets, while tending high rather than low.

A consistent trend among PIV test entries emerges. From the setpoint 7 and 46 comparisons, the T1 entries are consistently 0.01 or 0.02 high in axial turbulent velocity. This will be seen in the other six datasets when compared against other test entry data later. Subsequently, data from the T1 test being will be weighed less in the averaging process used to create the final Consensus datasets.
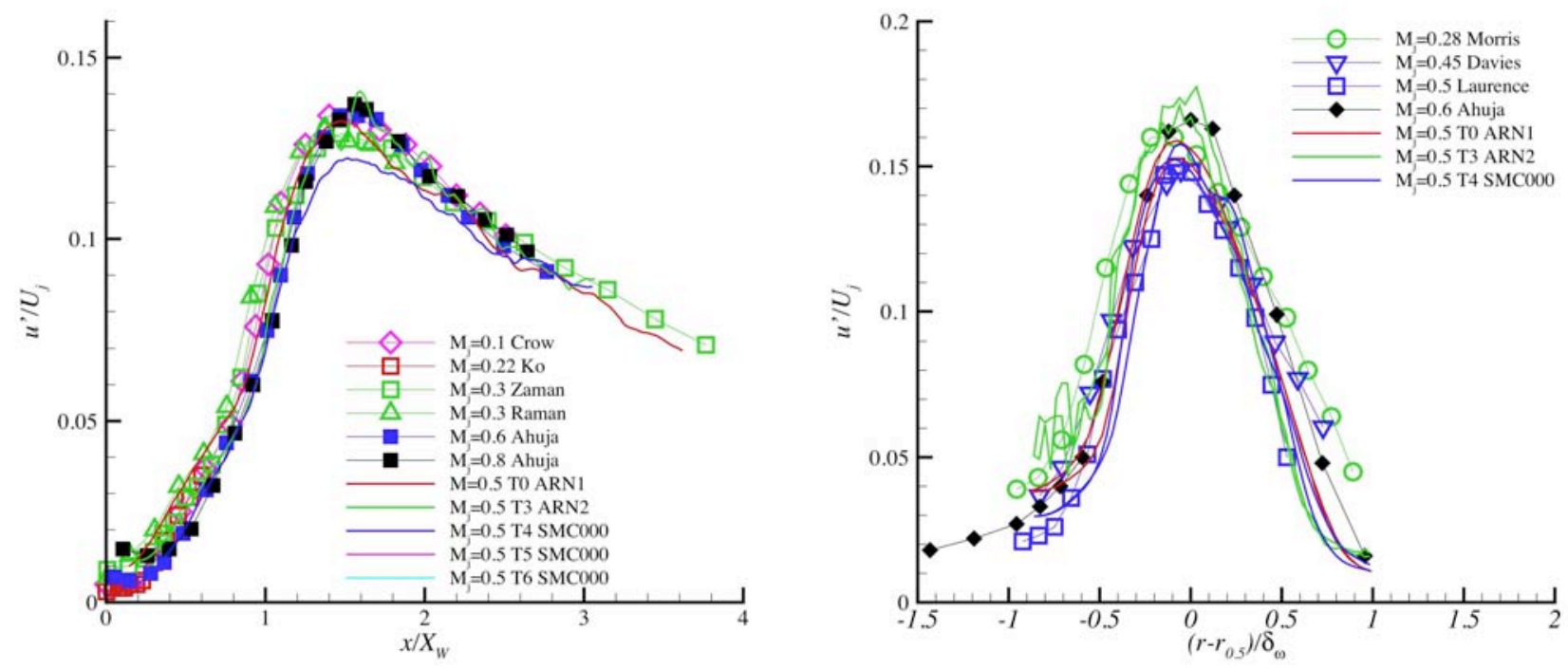

Figure 10 Plots of axial turbulent velocity ( $\mathrm{rms})$, in axial profile along centerline (left) and in radial profile at $x / D_{j}=4$ (right); low subsonic cold jet: (setpoint 3) $M a=0.5$, unheated. 

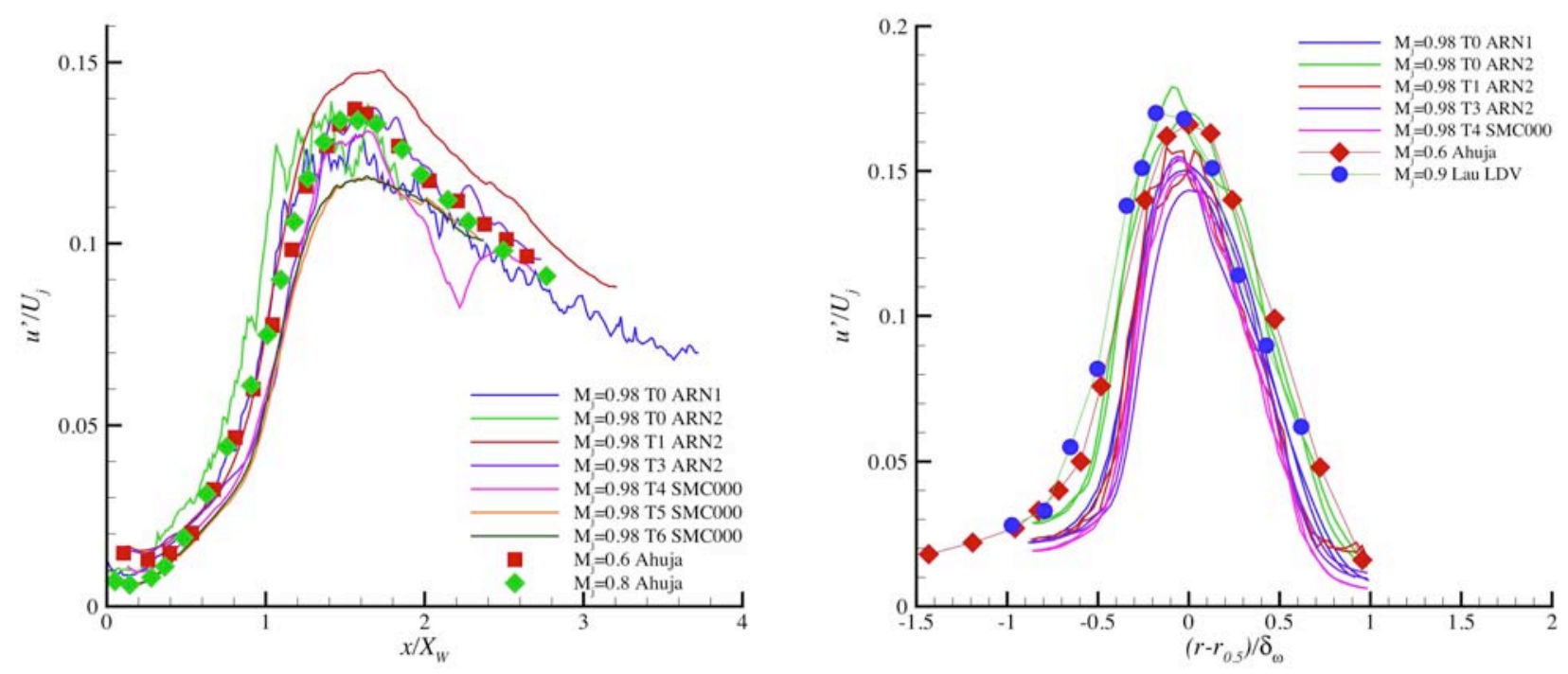

Figure 11 Plots of axial turbulent velocity (rms), in axial profile along centerline (left) and in radial profile at $x / D_{j}=4$ (right); high subsonic cold jet: (setpoint 7) $M a=0.9\left(M_{j}=0.98\right)$, unheated.
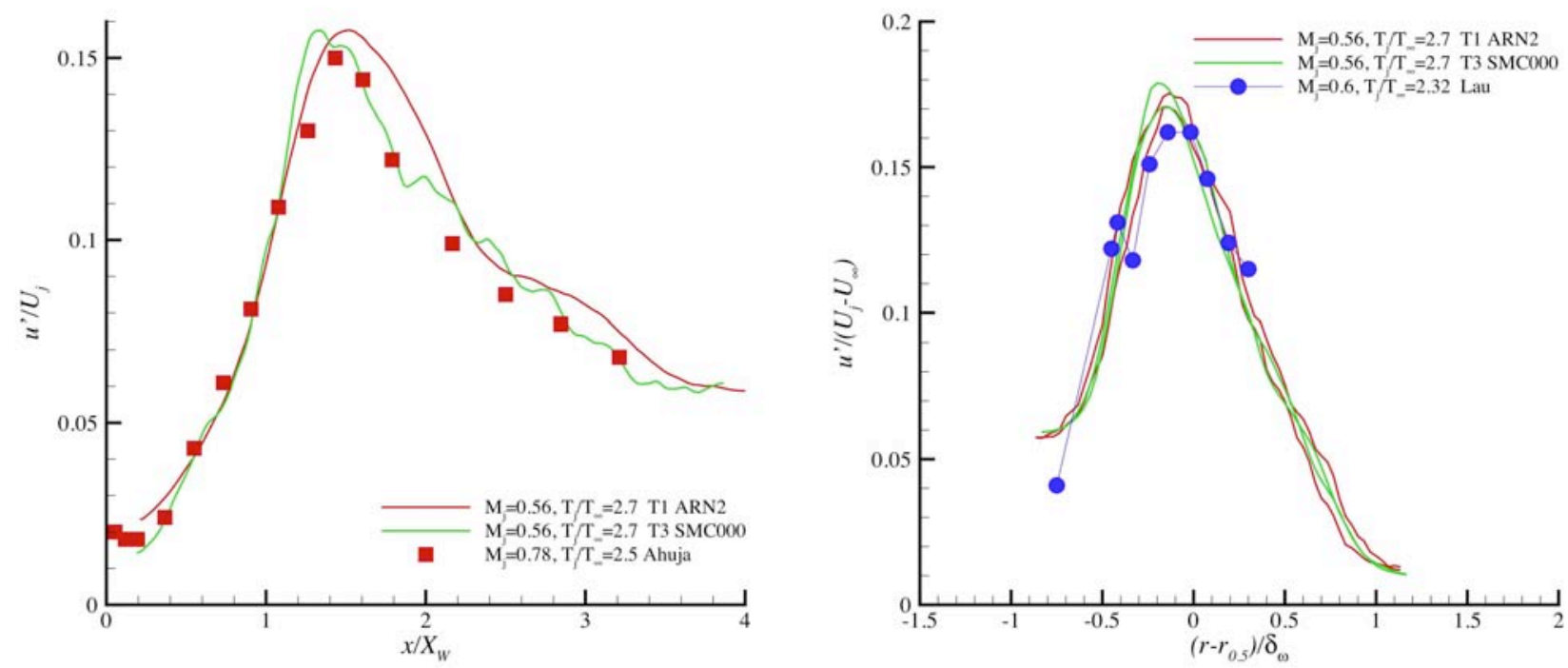

Figure 12 Plots of axial turbulent velocity (rms), in axial profile along centerline (left) and in radial profile at $x / D_{j}=4$ (right); high subsonic hot jet: (setpoint 46) $M_{j}=0.56$ at $T_{j} / T_{\infty}=2.7$. LDV data of Ahuja (Lepicovsky) for $M_{j}=0.78$, $T_{j} / T_{\infty}=2.54$ jet, LDV data of Lau 1980 for $M_{j}=0.6, T_{j} / T_{\infty}=2.32$.

When there is disparity among PIV datasets one should weigh the differing sets to arrive at a 'consensus' dataset, i.e., a weighted average of the measured data. One evaluation criterion is the amount of scatter within a single dataset, e.g. the amount of undulations on what should be a smooth profile. Some valuable information about the relative quality of the data can be arrived at by considering the internal checks on the data that occurred during processing of the PIV images. For instance, one metric used to identify erroneous correlations of particle images is the ratio of peak image correlation to the second-highest peak value. During PIV processing this is checked against a threshold and when the value is too low, the velocity vector is flagged as bad and not used in computing statistics of the flow. The number of valid velocity values at a point relative to the number of image pairs available is one measure of the 'quality' of the statistic derived from these data.

In theory the 'quality' metric should identify the least accurate datasets. However, as seen in Figure 13, this is not the case when comparing data from different test entries. The least smooth dataset is the T1 ARN2 set (green line) that differs most from the hotwire data, making it most suspect of the PIV datasets. However, as seen in the corresponding plot of 'quality' for these data, the T1 ARN2 set shows a very good quality metric compared with other PIV datasets. The worst quality was from the T4 SMC000 dataset (Figure 13, purple line) that agrees quite nicely with the hotwire and is very smooth, attributes that 
we look for in trusting data. Unfortunately, the quality metric is not consistent from one test entry to another, and cannot be used in an absolute sense to judge accuracy between datasets. It is, however, a good indicator of poor data within a given dataset. For example, consider the cases shown in Figure 14 where a portion of the T4 SMC000 dataset from $15<x / D_{j}<20$ appears suspicious. The quality metric supports the suspicion, being significantly lower in this axial region. This justifies removing this section of data from a consensus average or from evaluation of CFD if this were the only dataset available.
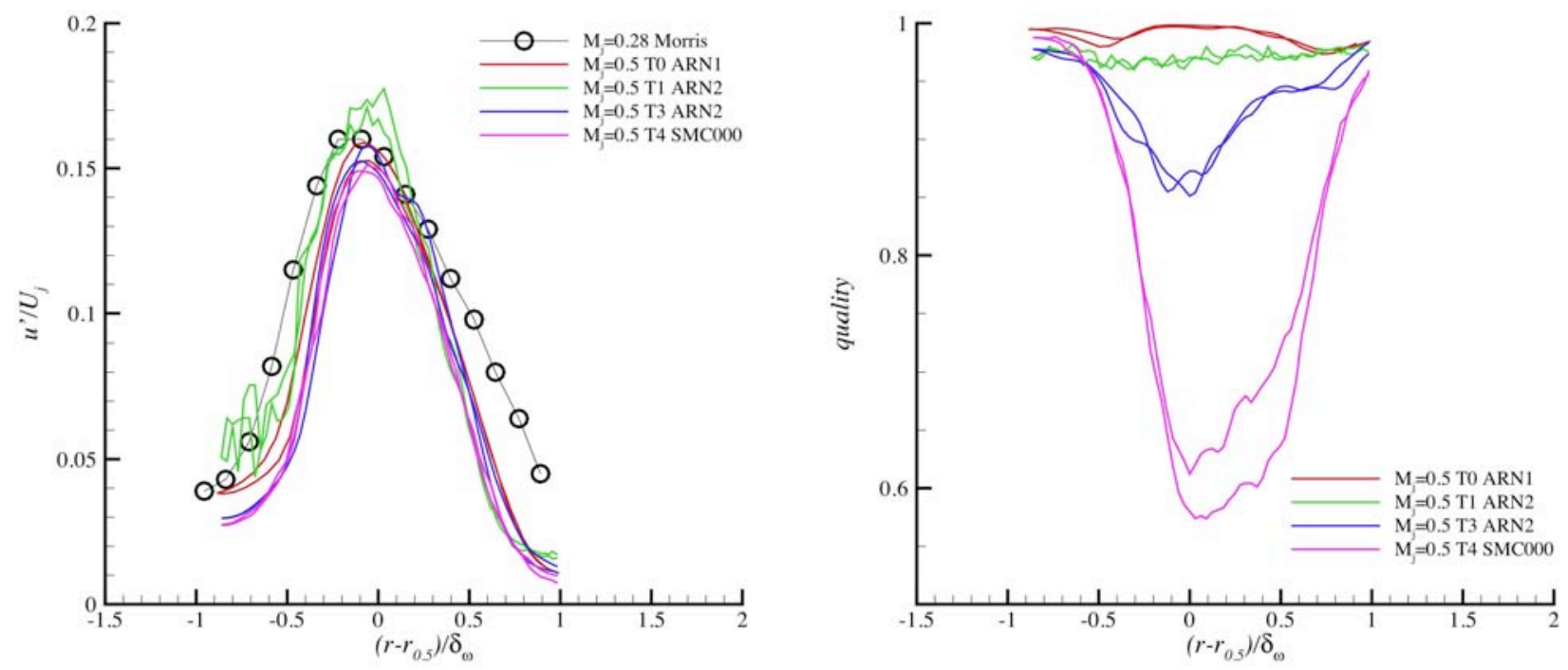

Figure 13 Attempting to use quality metric to weigh data between PIV datasets. Radial profile of turbulence measurements from PIV tests and their 'quality' metric (fraction of samples accepted in average) for the PIV data.
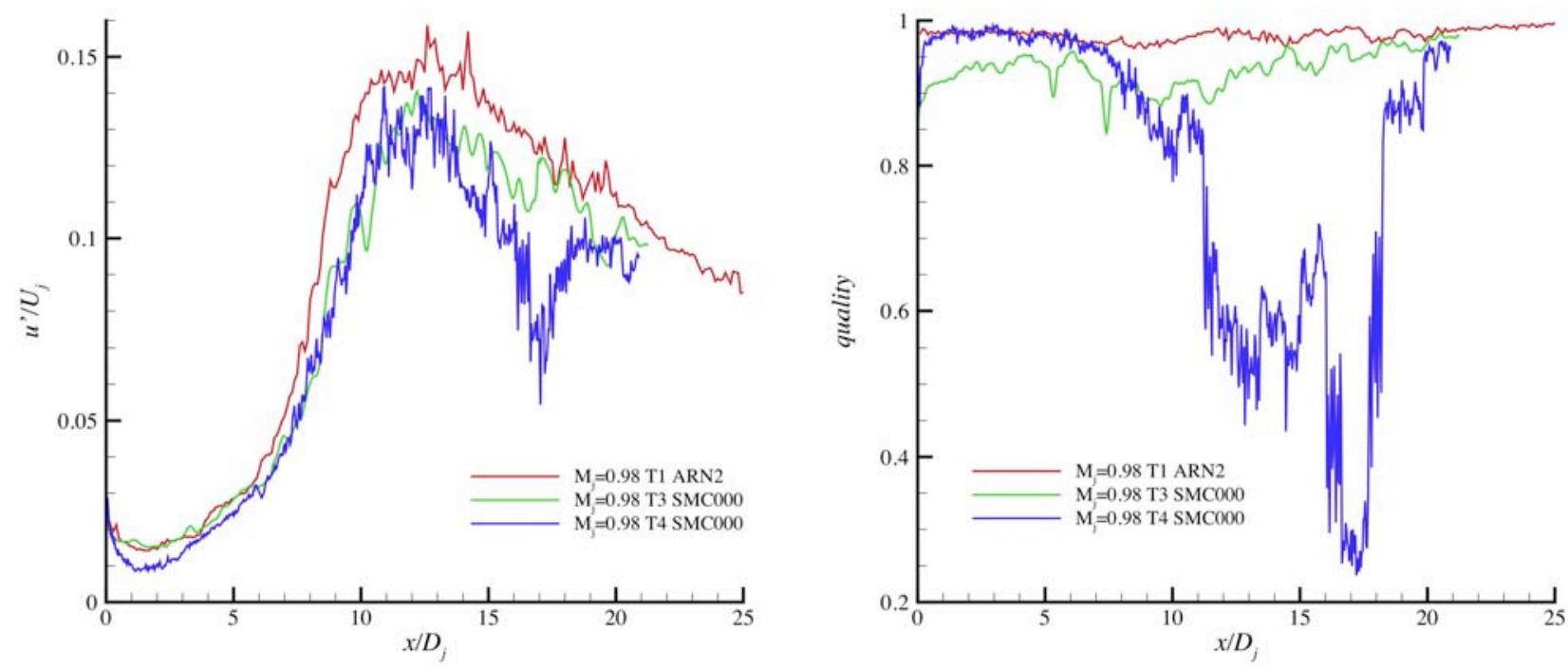

Figure 14 Example of using quality metric to confirm bad data within a dataset.

\section{The 'Consensus' Datasets}

For the convenience of the end user, it is desirable to produce a consensus dataset that represents the best estimate of the flow statistics, along with a measure of uncertainty. To do this, datasets for each setpoint were compared in axial and radial profiles. Where there were discrepancies, other factors such as the 'quality' metric and any field notes were studied. The smoothness of the data and its comparison to data from similar setpoints were considered. In some cases, primarily setpoint 7 , some datasets had to be excluded.

The actual averaging was done as follows. Each dataset was first smoothed in the axial direction using a moving average one diameter (20 samples) wide. The data was then interpolated onto a common grid of roughly the same spatial resolution as 
the original data, $0.02 \mathrm{D}_{\mathrm{j}}$ spacing axially and radially. The datasets for a given setpoint were then averaged point by point using the 'quality' metric to weight each sample. Finally, assuming axisymmetry, the two halves of the flow were averaged to produce a final, smoothed, averaged, symmetric dataset. Figure 15 and Figure 16 demonstrate this on the two-dimensional contour plot of axial turbulence intensity for setpoint 7, displaying individual datasets and the consensus dataset.

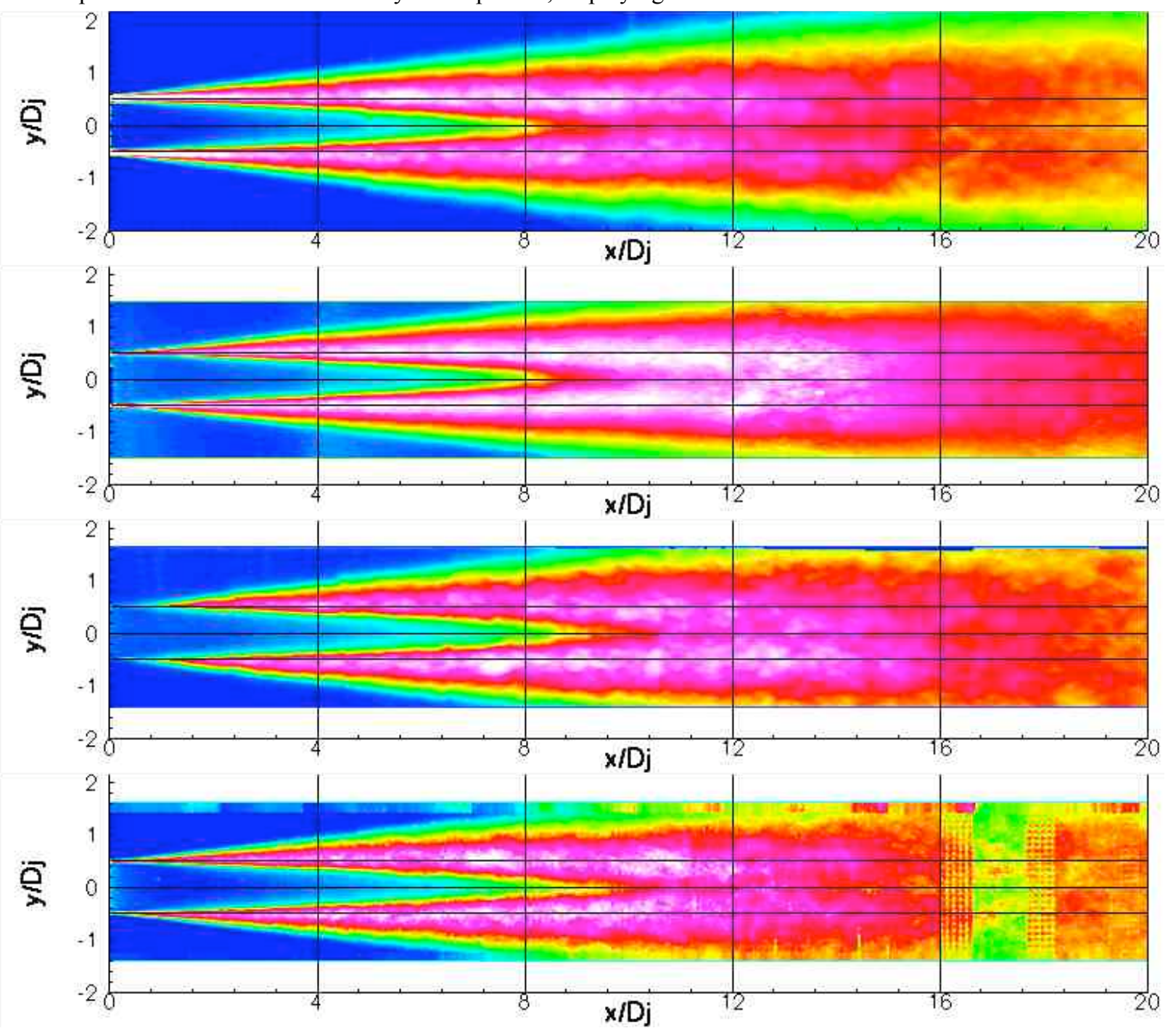

Figure 15 Reference figure for locating line plots extracted from tw-dimensional datasets. Contour plots of axial turbulence intensity for setpoint 7. From the top, test entries T0, T1, T3, and T4.

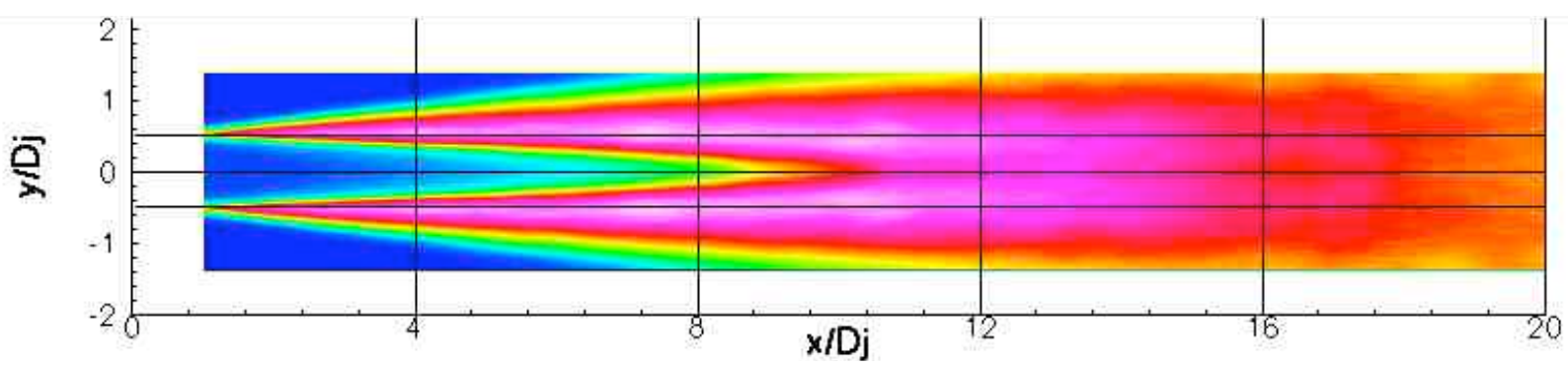

Figure 16 Consensus dataset for data from previous figure.

For detailed presentation, line plots from these datasets will be presented in a series of plots below. Line plots have been extracted from the input and final datasets along two axial lines $\left(x / D_{j} ; y / D_{j}=-0.5,0,+0.5\right)$ and along four radial lines $\left(x / D_{j}=\right.$ $\left.4,8,12,16 ; y / D_{j}\right)$. The lines where data are extracted are shown in Figure 15 for visual reference. Specifically, two figures 
are given for each setpoint. Four plots in the first figure show axial profiles of mean and turbulent axial velocity on the centerline and lipline. Eight plots in the second figure show radial profiles of mean and turbulent axial velocity at the four axial stations.

Starting with setpoint 3 (Figure 17 and Figure 18), a low dispersion among the datasets is observed for the centerline data: the scatter band is less than 0.02 for $U / U_{j}$ and around 0.02 for $u^{\prime} / U_{j}$. There is a more scatter among the datasets along the lipline, especially for the mean velocity with a scatter band of 0.05 downstream of $x / D_{j}=5$, and worse upstream near the nozzle exit. Small discrepancies in the radial registration of the datasets were a prime contributor to this discrepancy near the nozzle lip. In the radial profiles (Figure 18), the scatter band is closer to that of the centerline axial profile.
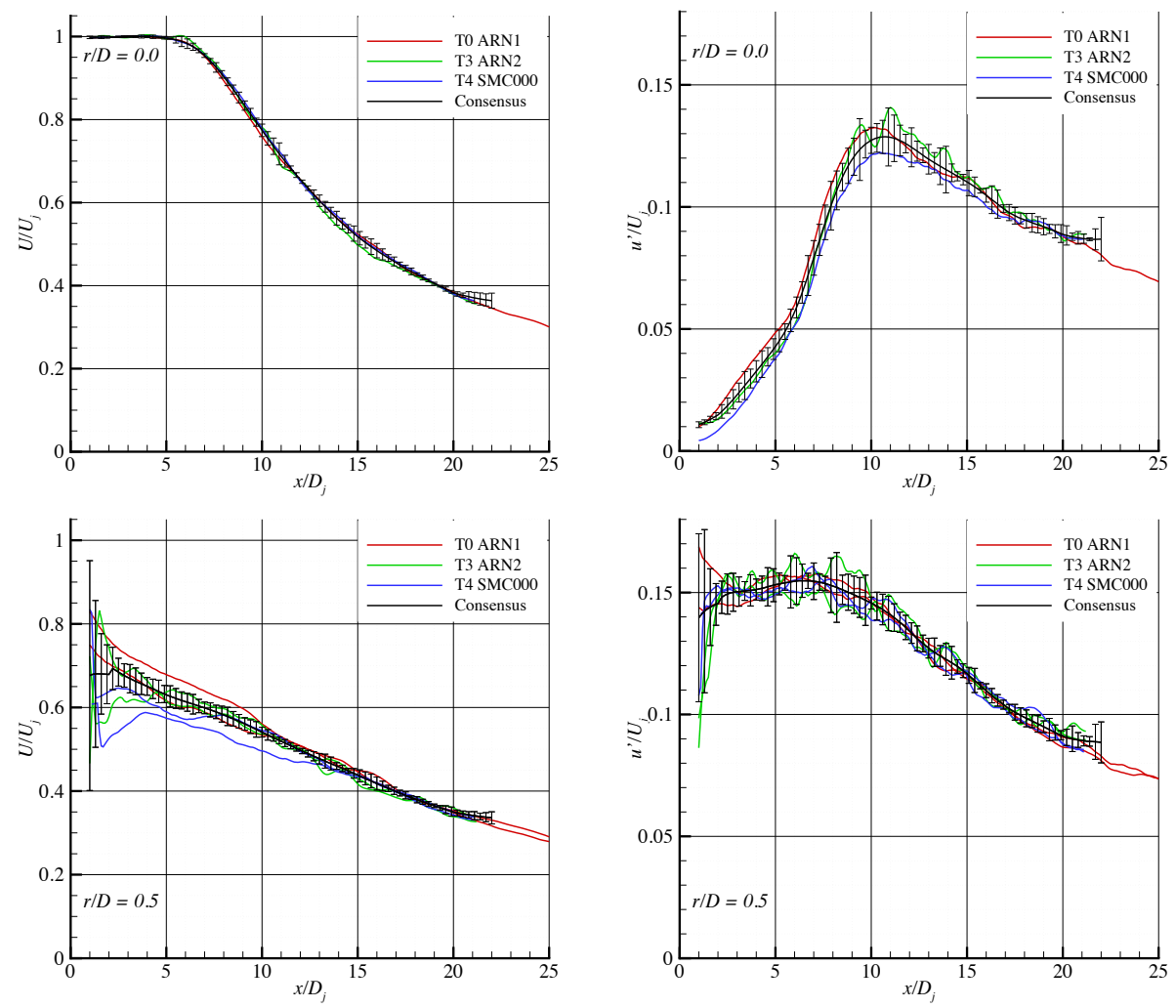

Figure 17 Setpoint 3: Axial profiles, axial mean and turbulent velocities on the centerline (top) and on the lipline (bottom). 

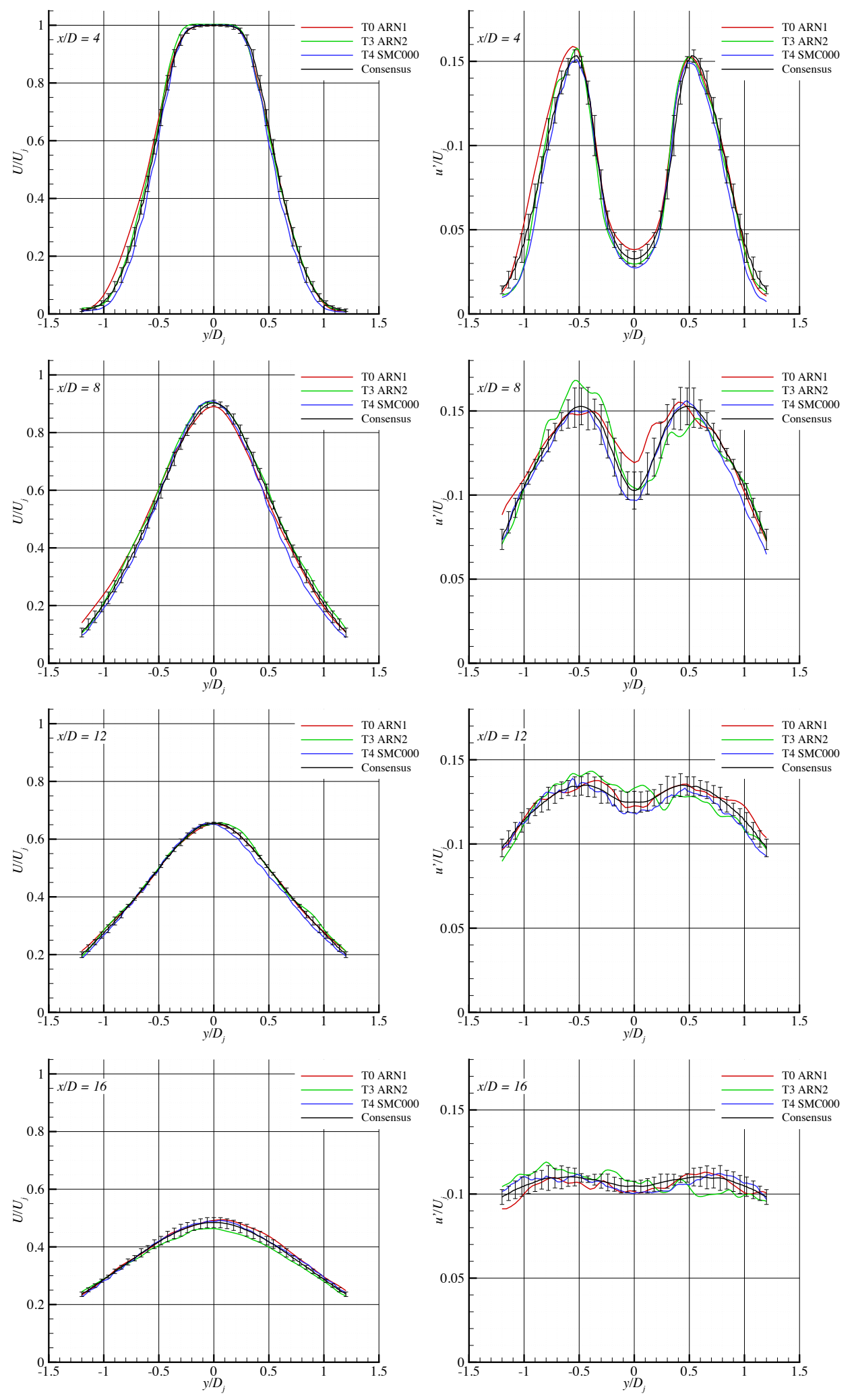

Figure 18 Setpoint 3: Radial profiles, axial mean and turbulent velocities at $x / D_{j}=4,8,12$, and 16 .

Setpoint 7 was a frustrating case. Although the T1 ARN2 dataset is shown in the figures, only T3 ARN2 and T4 SMC000 were used in the consensus dataset. An unexpected rise in turbulence on the centerline at the end of the potential core of the 
T1 ARN2 dataset seemed too suspect, especially when this dataset was compared against many other datasets, normalized for potential core length. This left only 20 diameters of valid data for the consensus dataset. Another affliction was the region from $15<x / D_{j}<20$ in the T4 SMC000 dataset, discussed previously. This left only the T3 ARN2 dataset to be used in this region and some irregularities in the axial profiles from jumping between datasets. The agreement between these two datasets in their valid region was quite good, however, with uncertainties similar to that of the setpoint 3 dataset.
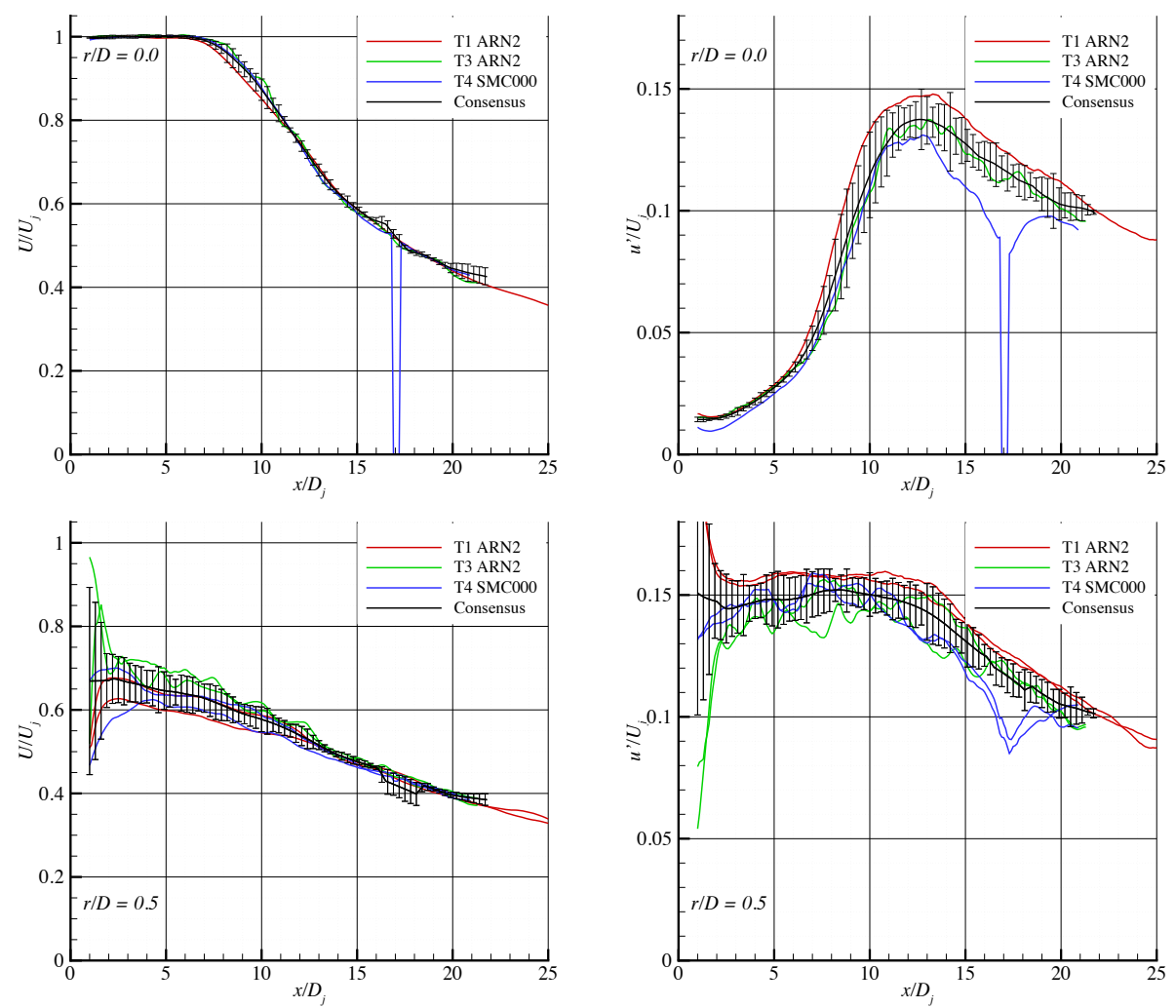

Figure 19 Setpoint 7: Axial profiles, axial mean and turbulent velocities on the centerline (top) and on the lipline (bottom). 

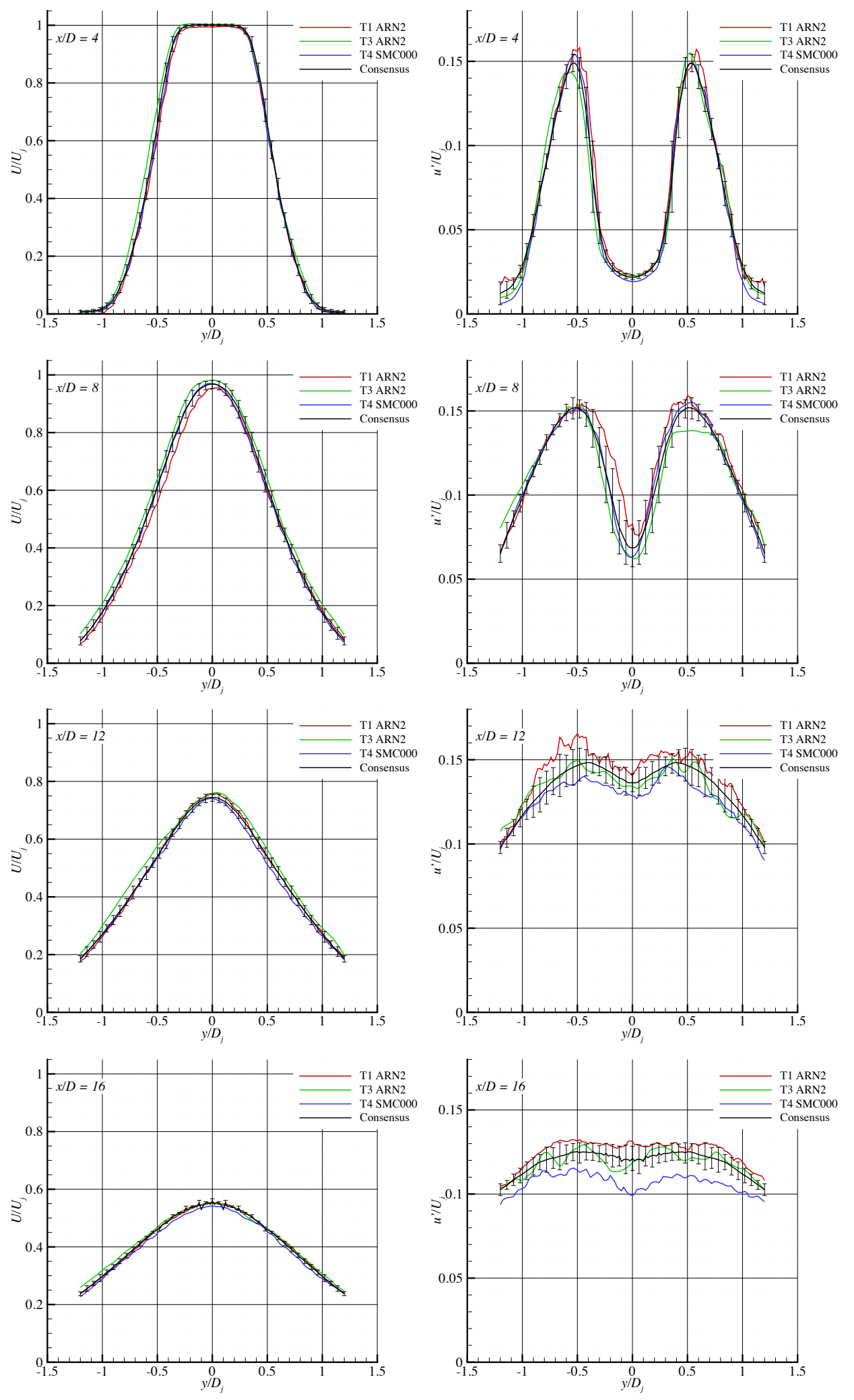

Figure 20 Setpoint 7: Radial profiles, axial mean and turbulent velocities at $x / D_{j}=4,8,12$, and 16 .

Setpoints 23, 27, 29, and 46, (Figure 21 - Figure 28) all had only two datasets upon which to base a consensus dataset. Luckily, these datasets had no significant internal quality issues. The turbulence levels differed by as much as 0.02 in $u^{\prime} / U_{j}$ 
between the two datasets. The consensus datasets, therefore, generally split the difference and should be considered to have a larger uncertainty than the two cold setpoints. It does not seem that temperature is the prime culprit, but this discrepancy is consistent across all four setpoints.
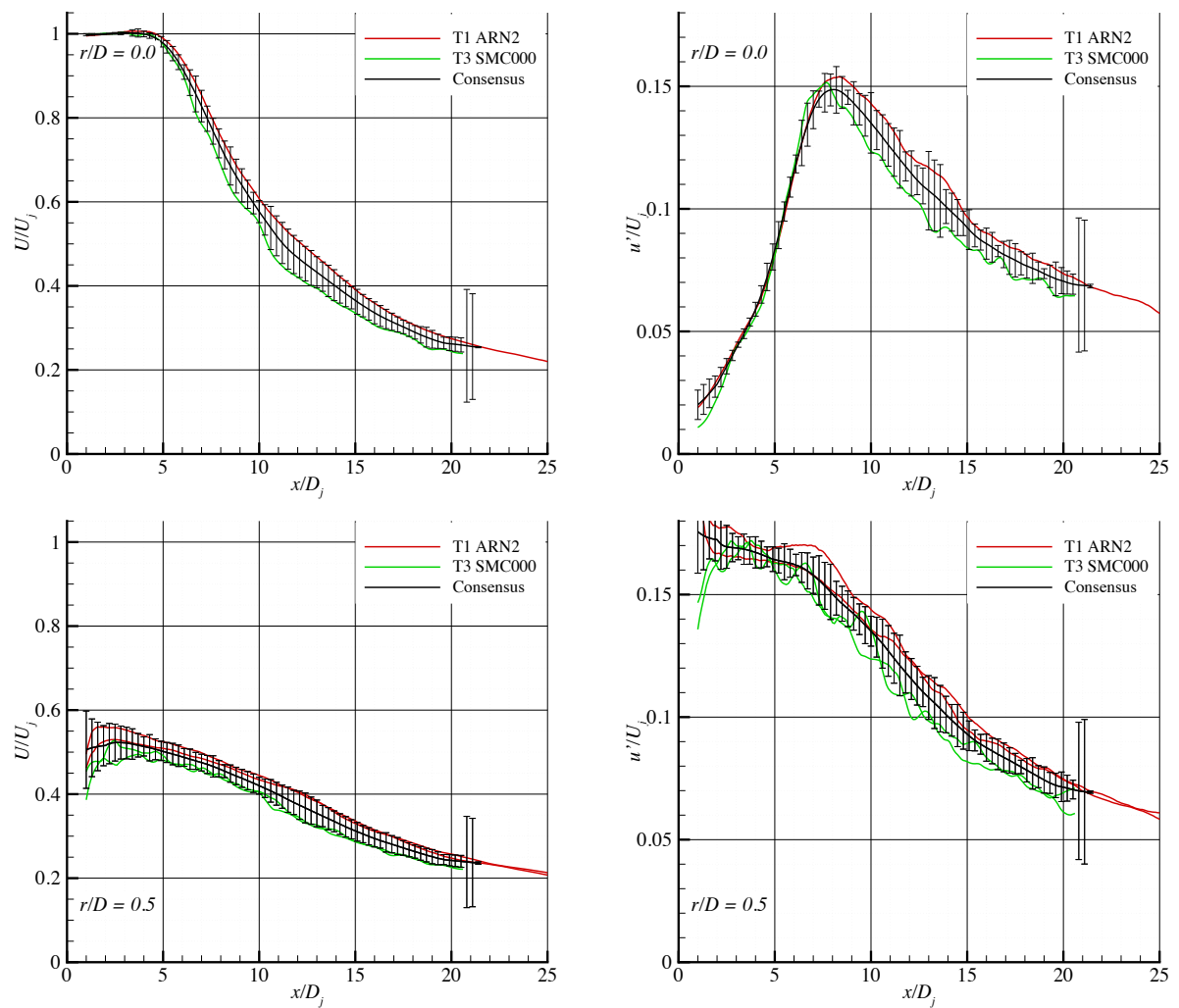

Figure 21 Setpoint 23: Axial profiles, axial mean and turbulent velocities on the centerline (top) and on the lipline (bottom). 

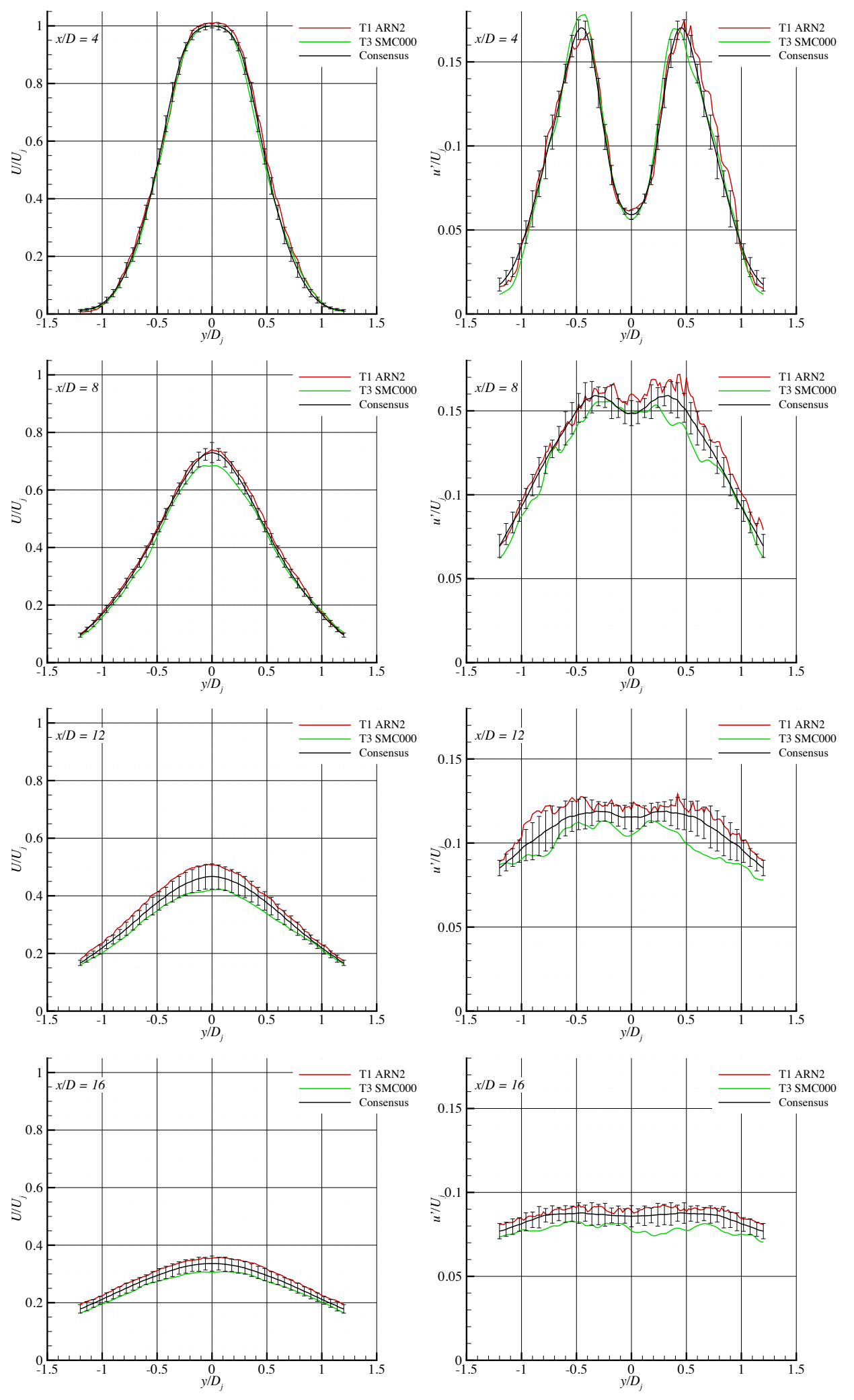

Figure 22 Setpoint 23: Radial profiles, axial mean and turbulent velocities at $x / D_{j}=4,8,12$, and 16 . 

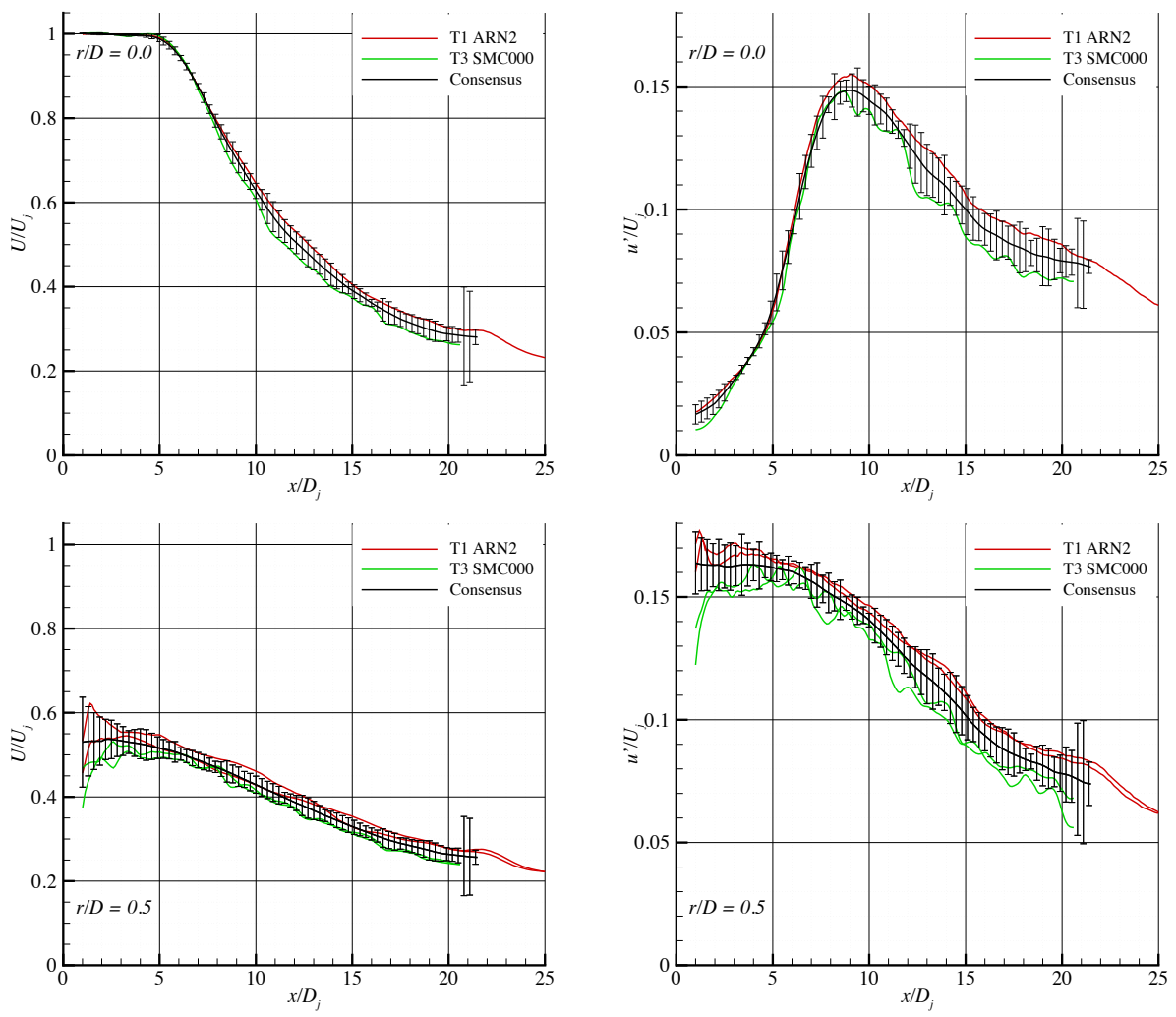

Figure 23 Setpoint 27: Axial profiles, axial mean and turbulent velocities on the centerline (top) and on the lipline (bottom). 

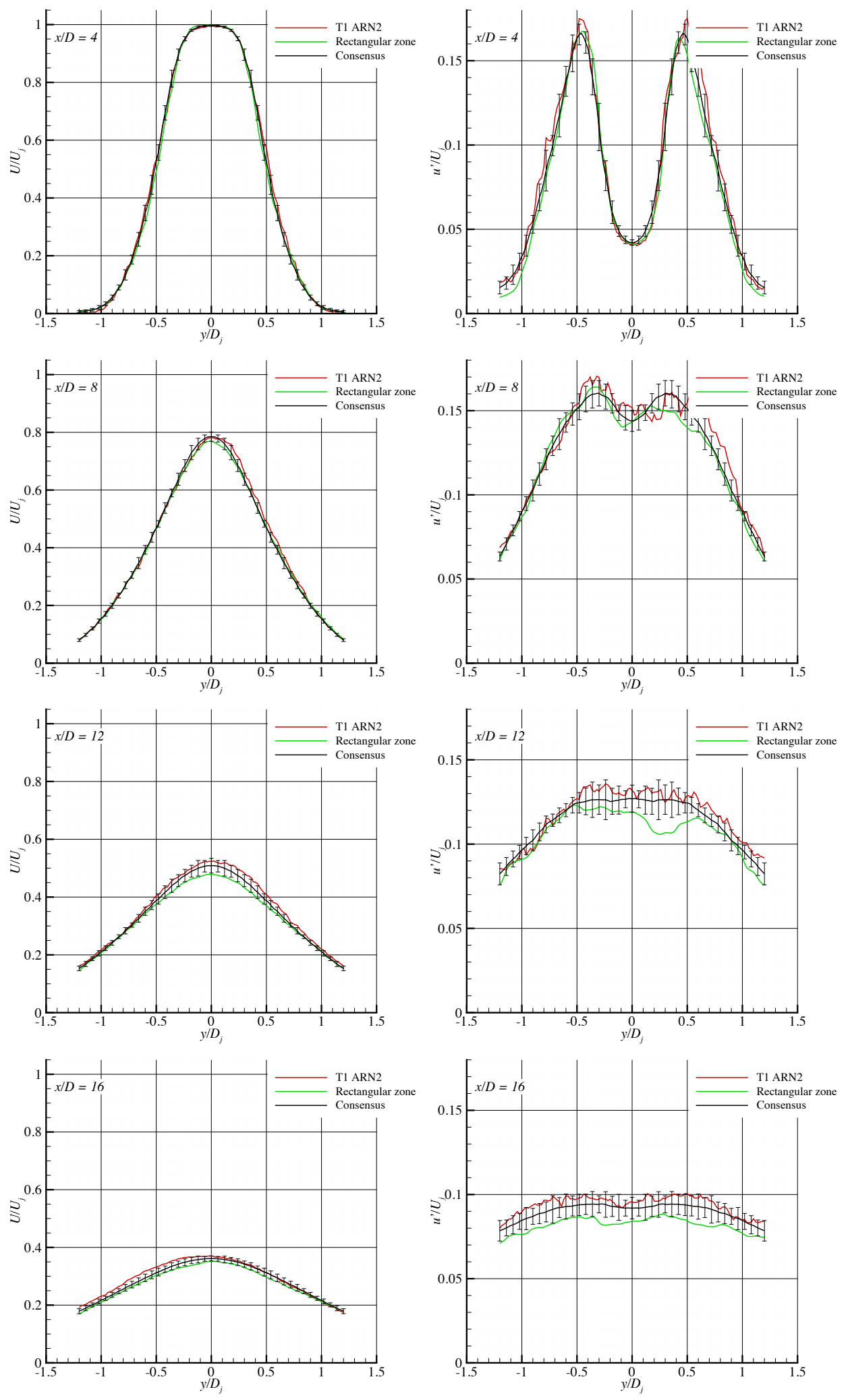

Figure 24 Setpoint 27: Radial profiles, axial mean and turbulent velocities at $x / D_{j}=4,8,12$, and 16 . 

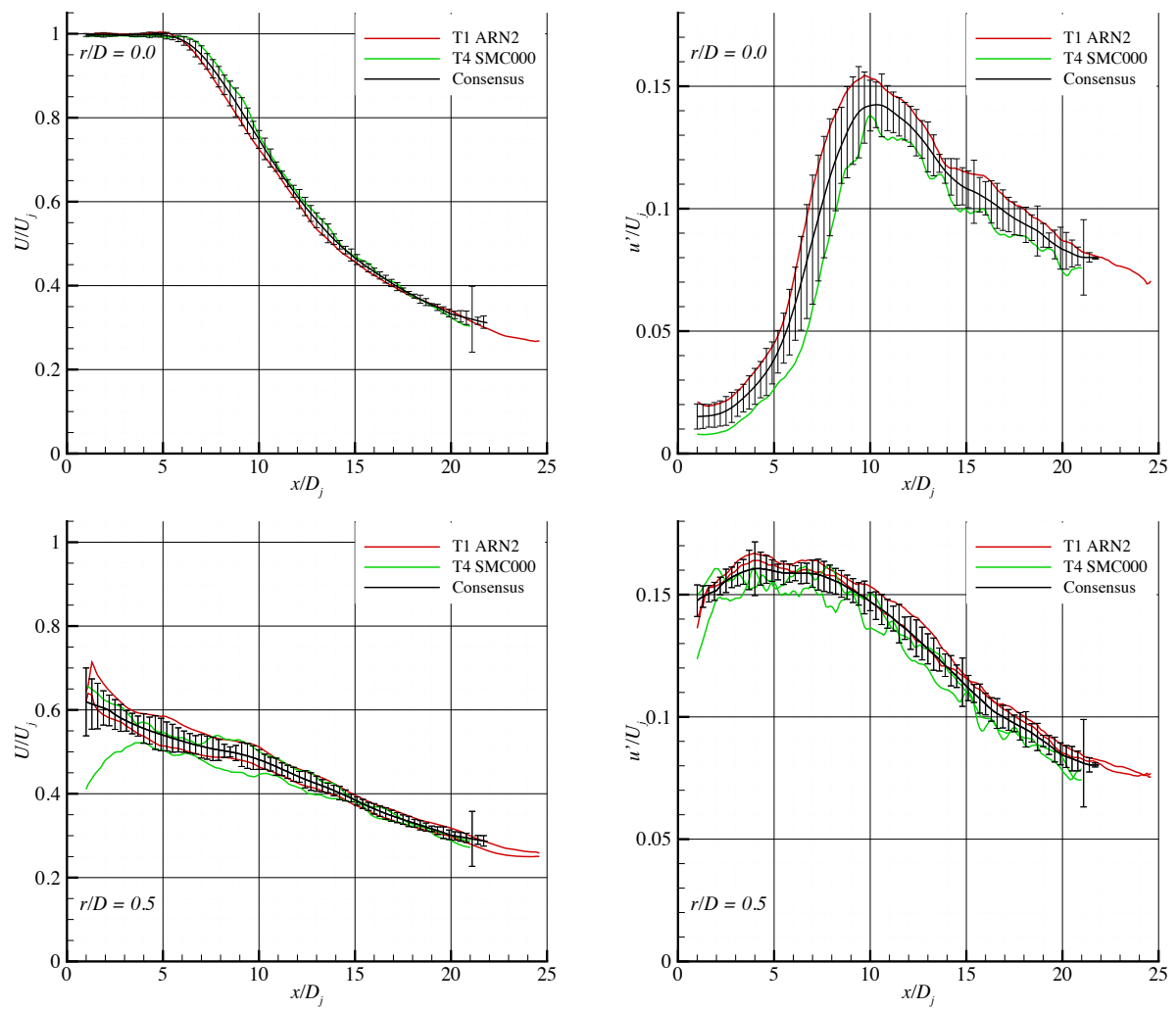

Figure 25 Setpoint 29: Axial profiles, axial mean and turbulent velocities on the centerline (top) and on the lipline (bottom). 

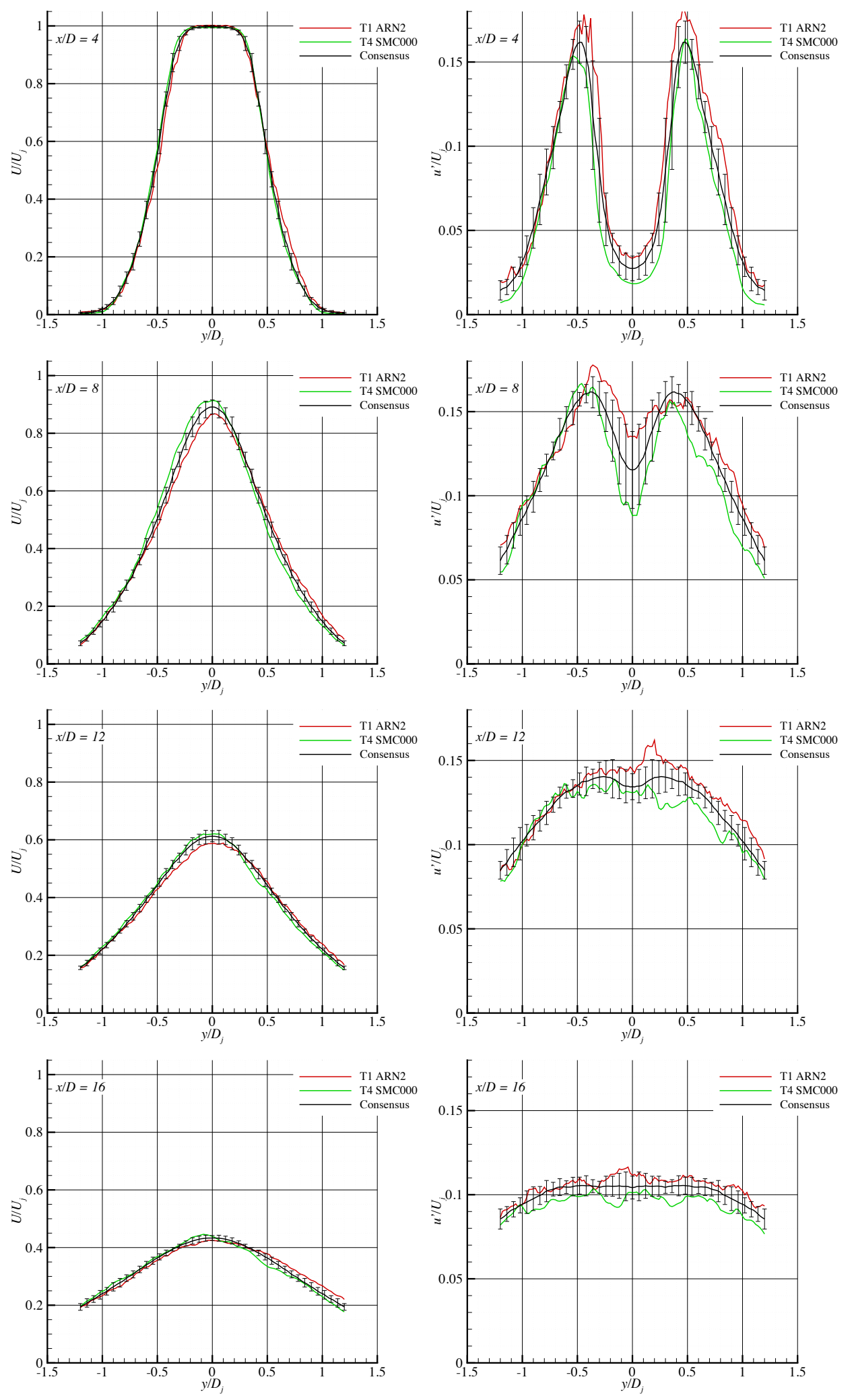

Figure 26 Setpoint 29: Radial profiles, axial mean and turbulent velocities at $x / D_{j}=4,8,12$, and 16 . 

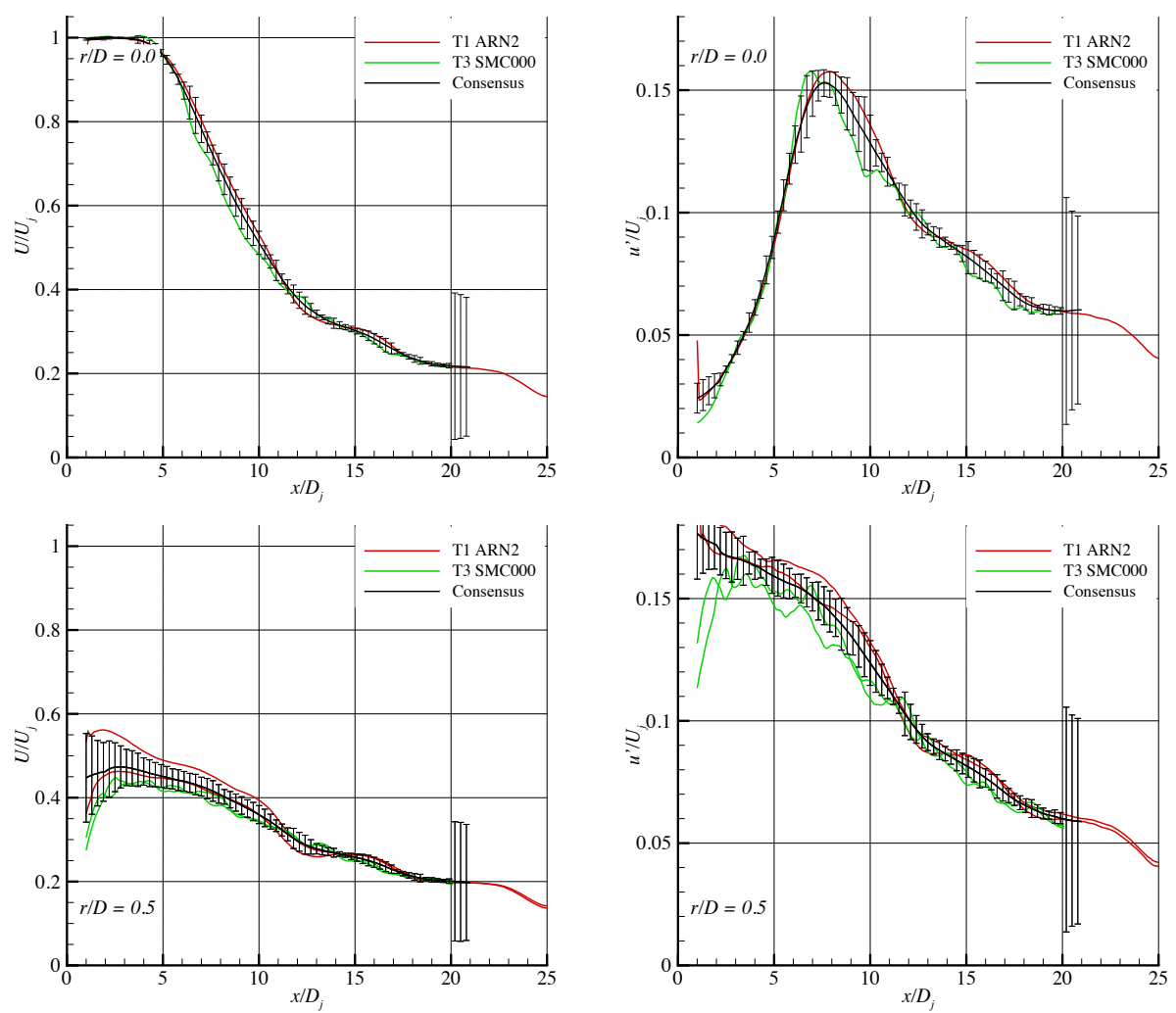

Figure 27 Setpoint 46: Axial profiles, axial mean and turbulent velocities on the centerline (top) and on the lipline (bottom). 

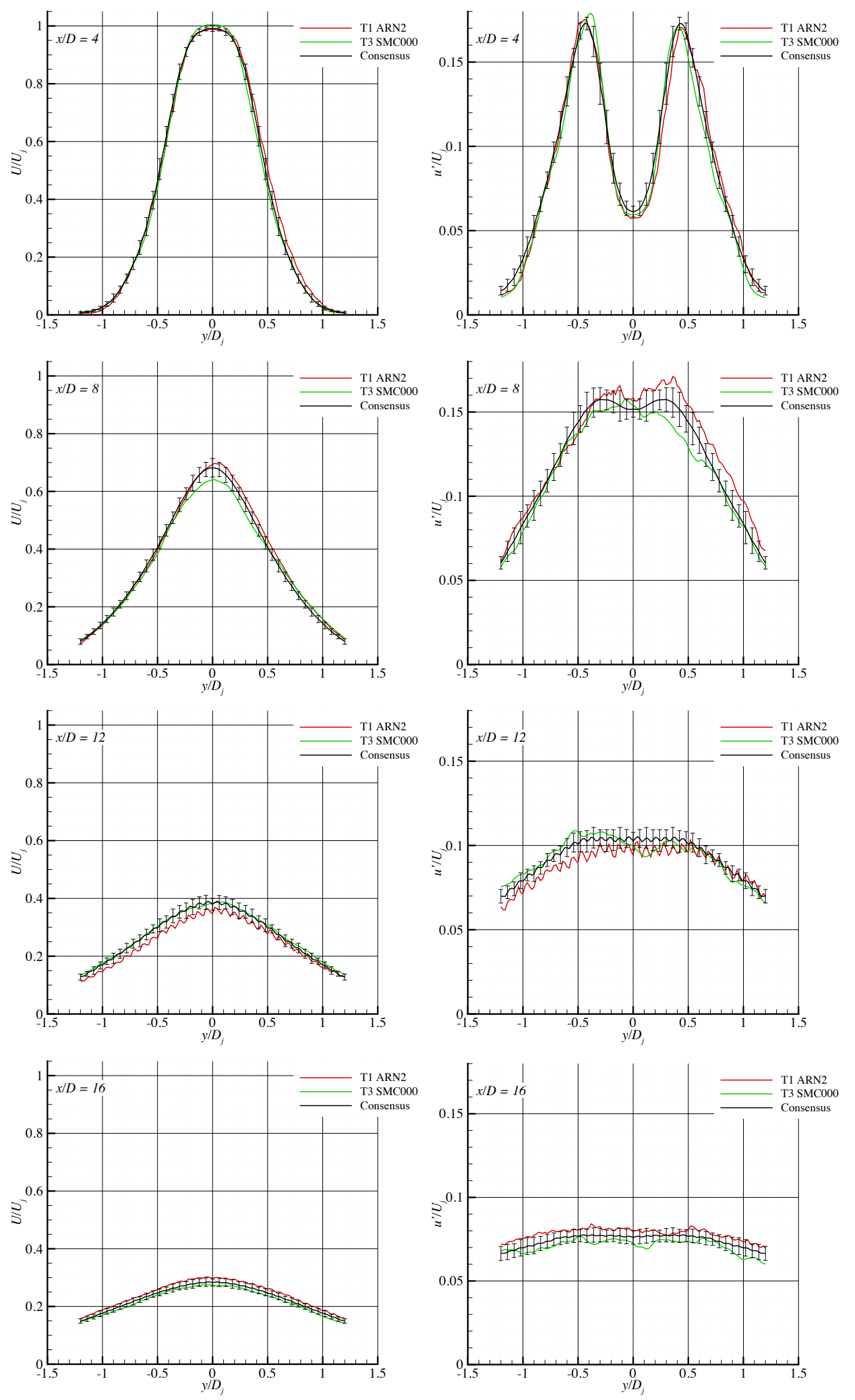

Figure 28 Setpoint 46: Radial profiles, axial mean and turbulent velocities at $x / D_{j}=4,8,12$, and 16 . 

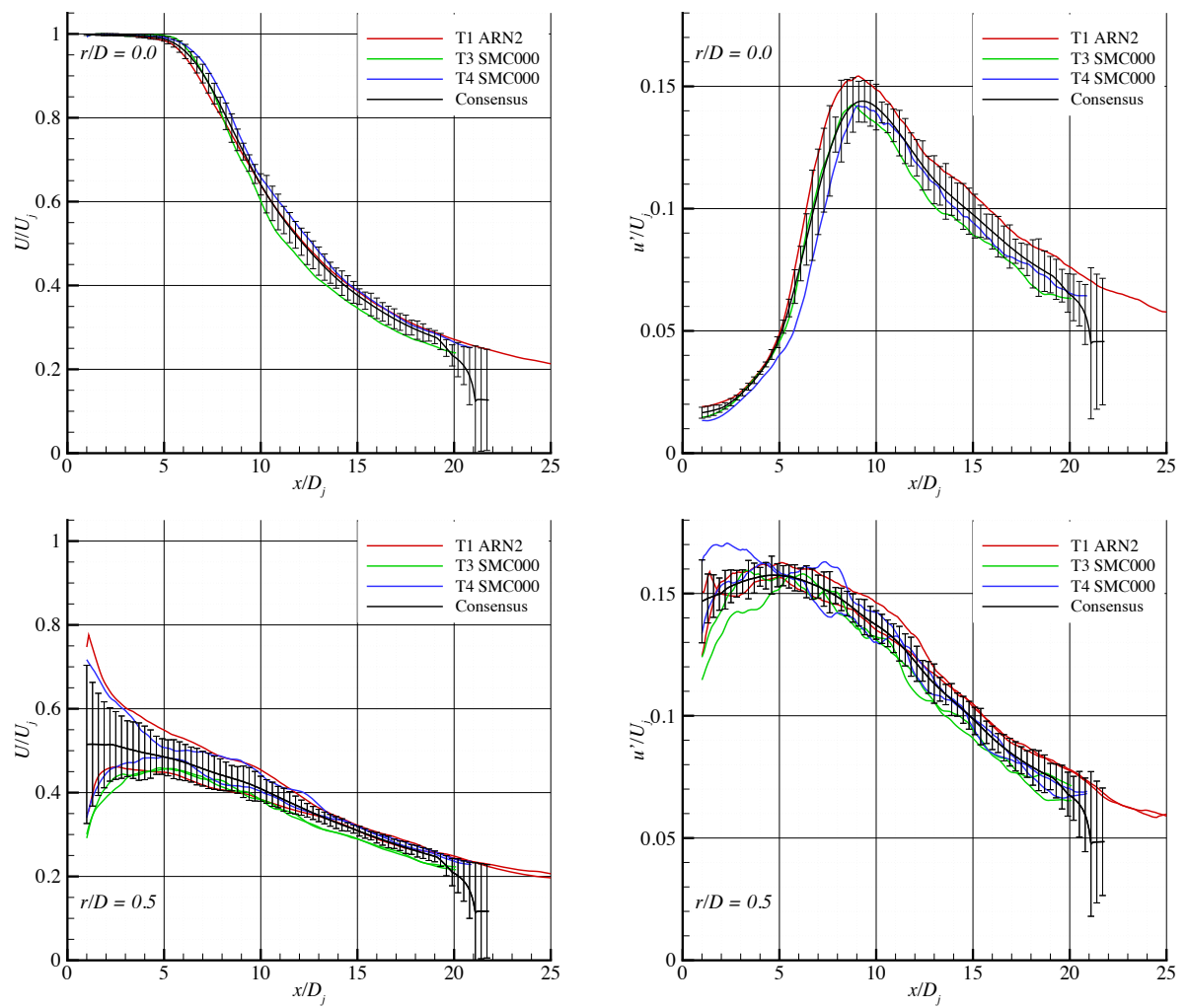

Figure 29 Setpoint 49: Axial profiles, axial mean and turbulent velocities on the centerline (top) and on the lipline (bottom). 

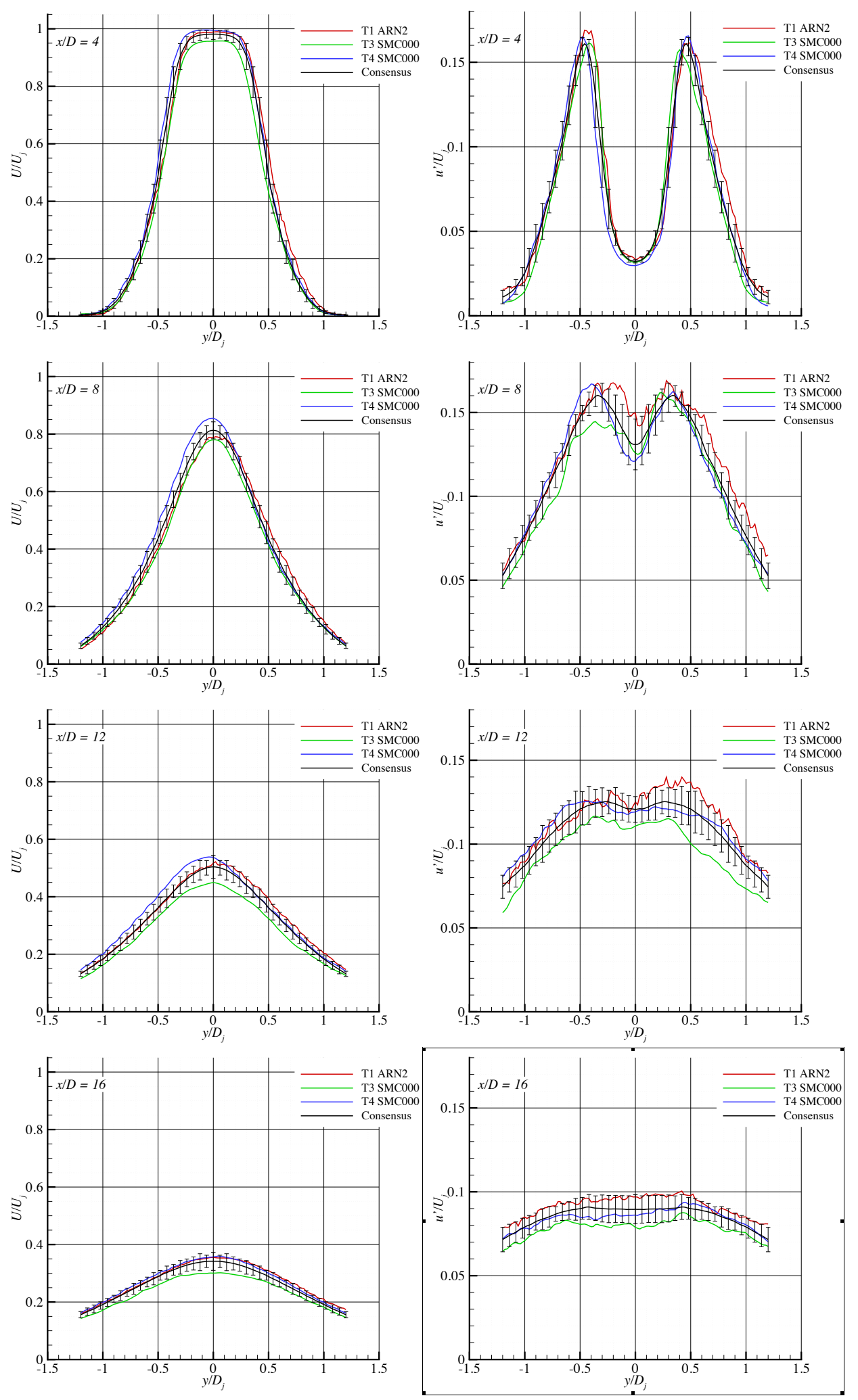

Figure 30 Setpoint 49: Radial profiles, axial mean and turbulent velocities at $x / D_{j}=4,8,12$, and 16 .

Returning to the data from literature, the Consensus datasets are plotted over the data from literature presented earlier. The low subsonic cold jet data of Figure 2 is repeated in Figure 31 with the setpoint 3 Consensus dataset added. No 
significant bias is evident from this plot. Similarly, the high subsonic cold jet data of Figure 3 is repeated in Figure 32 with the setpoint 7 Consensus dataset added. Here, depending upon whether the LDV data is included in the literature data, the Consensus data has either no bias (including LDV) or a +0.01 bias in peak axial turbulence intensity. And finally, LDV data from Lau measured in a $M_{j}=0.6, T_{j} / T_{\infty}=2.32$ subsonic hot jet is compared with the setpoint 46 Consensus data, where it is found to agree to within the unevenness of the LDV data. There might be a bias of +0.005 , but there is really not enough data to make such a fine distinction.

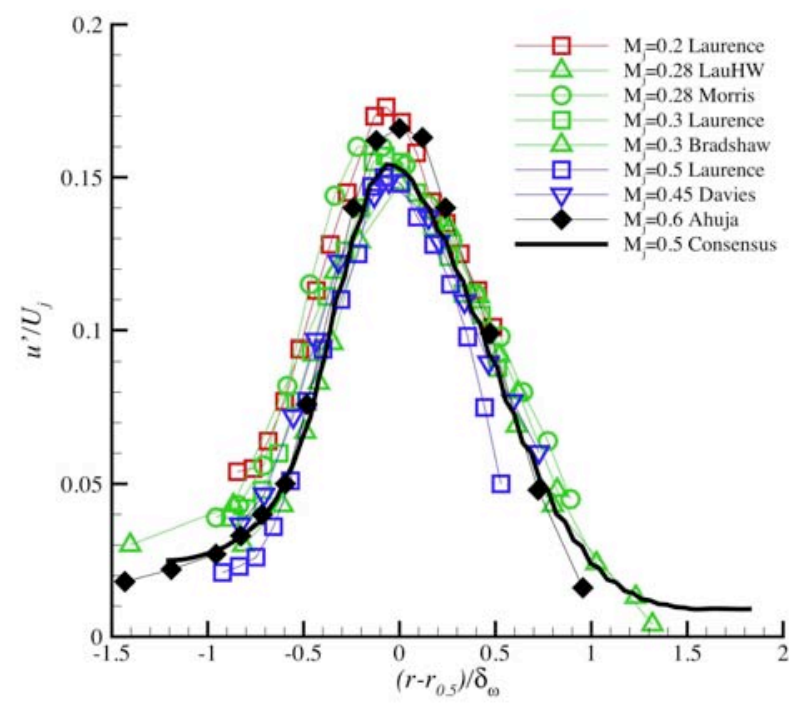

Figure 31 Radial profiles of axial turbulence at $x / D_{j} \sim 4$ for low subsonic, cold jets (setpoint 3). Consensus data vs validated literature data.

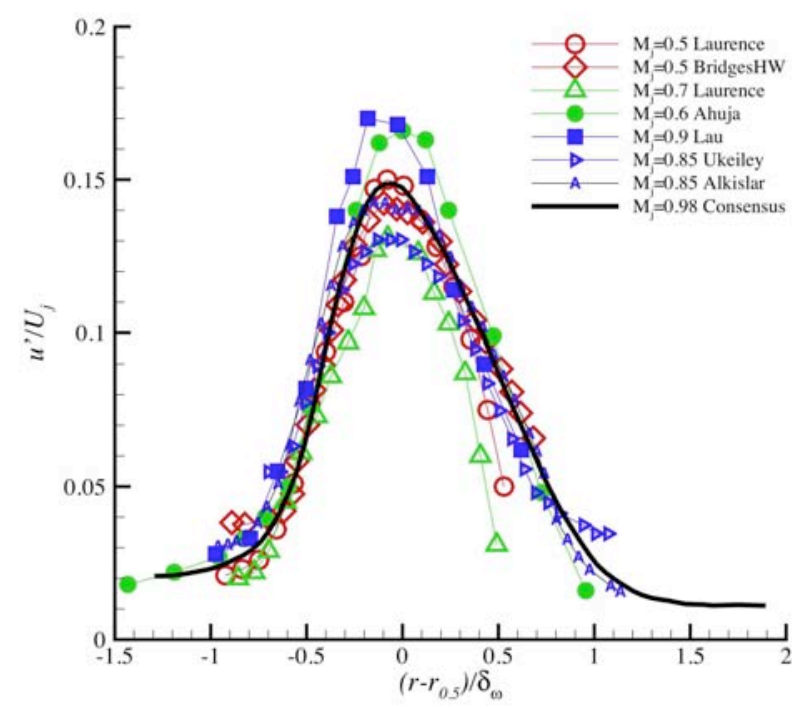

Figure 32 Radial profiles of axial turbulence at $x / D_{j} \sim 4$ for high subsonic, cold jets (setpoint 7 ). Consensus data vs validated literature data. 


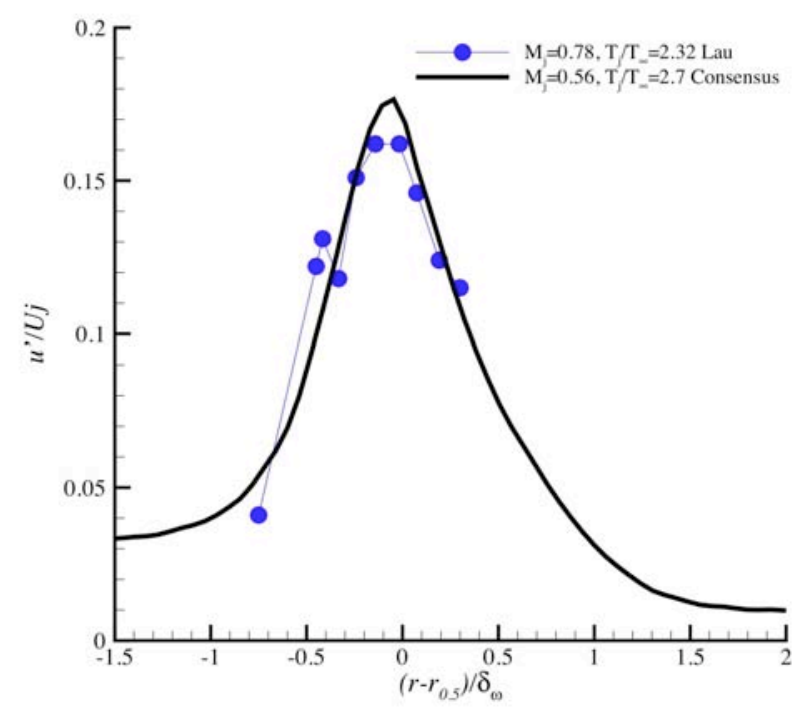

Figure 33 Radial profiles of axial turbulence at $x / D_{j} \sim 4$ for high subsonic, hot jets (setpoint 46 ). Consensus data vs validated literature data.

\section{Discussion}

\section{A. Uncertainties}

One of the most overlooked questions in most experimental work is the question of accuracy. Although most journals require some generally accepted statement of accuracy, the majority of papers do not quantify (and justify!) their uncertainties. Having created error estimates for the jet flow data at many speeds and temperatures, it is worthwhile to bring these estimates together and to establish a general estimate for uncertainty of other PIV measurements acquired with the same degree of care in a similarly qualified facility.

In Figure 34 all the uncertainties shown by error bars in the Consensus dataset plots above have been gathered as line plots. The plots show the standard deviations of the mean and turbulence measurements along both centerline and lipline. Uncertainties in the mean velocity are strongly associated with the gradients in the flow. Along the centerline (top left plot), the uncertainty in normalized mean velocity $U / U_{j}$ is $2 \%$ to $4 \%$, independent of axial position. Along the lipline of the nozzle (bottom left plot) the uncertainty is a very strong function of axial position. The significant increase in uncertainty near the nozzle is mostly related to registration between datasets as the shear layer is of the order of the measurements' spatial resolution. Beyond five diameters the uncertainty is again less than $4 \%$, similar to the centerline.

The uncertainties in turbulence measurements are proportional to the turbulence intensities. Along the centerline (top right plot) the uncertainty in most datasets registers below 0.01 , or roughly $7 \%$ of the peak value $\left(u^{\prime} / U_{j}=15 \%\right)$. A few of the datasets show uncertainties closer to 0.02 near the peak. Along the lipline all cases show uncertainty below 0.01 downstream of $x / D_{j}=2$. Closer to the nozzle the uncertainty is higher, as expected due to the lack of sufficient resolved points within the thin shear layer. 

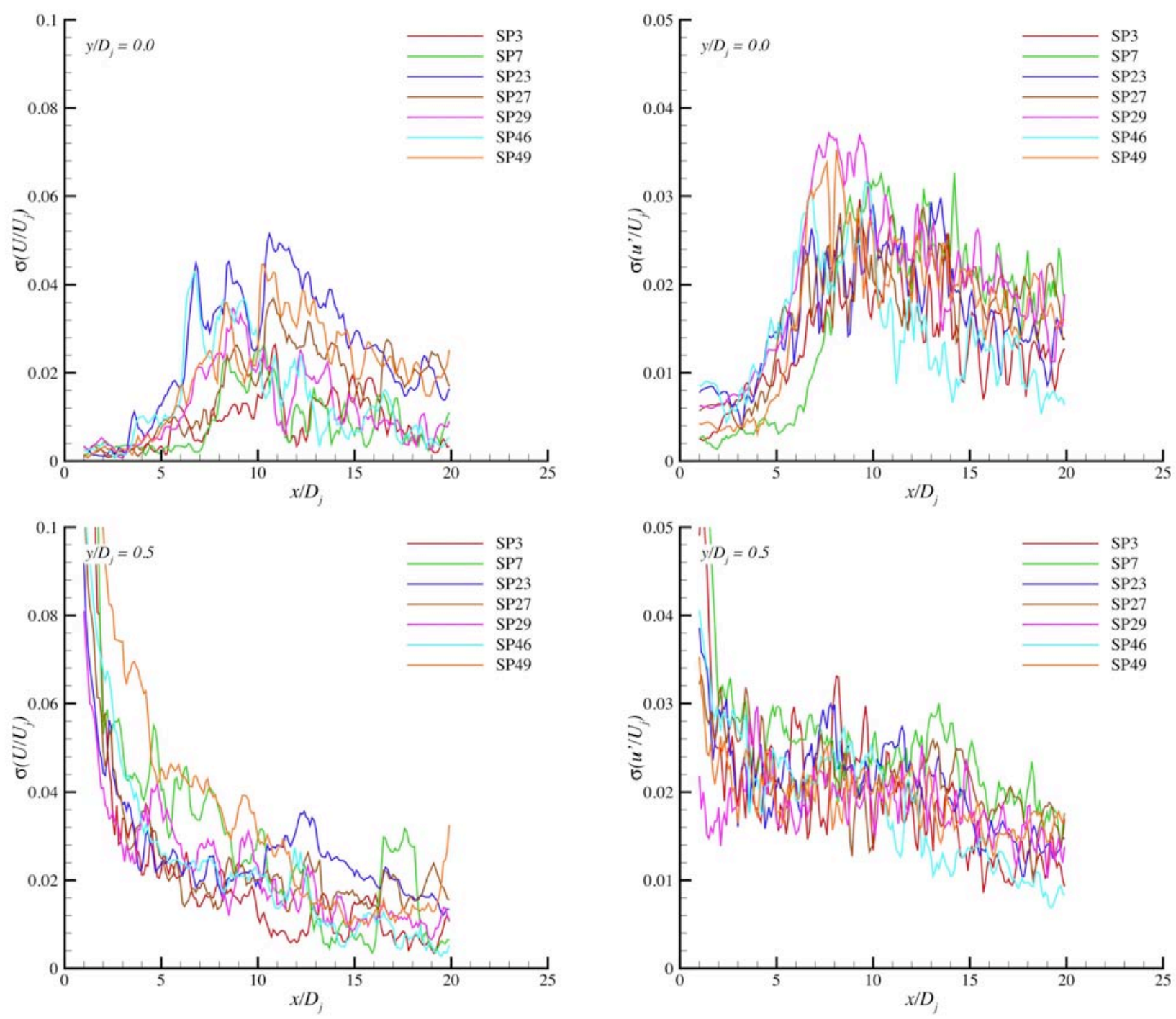

Figure 34 Uncertainties in mean (left) and turbulent (right) axial velocities for all setpoints along the jet centerline (top) and lipline (bottom).

\section{B. Effect of Initial Conditions}

In Section II.C above, hotwire measurements were presented for the initial jet shear layer, parameterized by boundary layer statistics. The data were acquired at low Mach numbers, up to $M a=0.3$, well within the limitations of the hotwire anemometer. Two concerns were raised that could impact the growth of the initial shear layer and the subsequent jet plume: (1) nozzle type (ARN vs SMC) and (2) rig flow conditioning (upstream screens before entry T4, none during T4 and afterwards). Of the data conditions acquired, the most sensitive to these changes would be the low Mach number flows, such as setpoint 3. While no two PIV datasets were acquired at setpoint 3 which isolated these concerns, datasets do exist which show how both changes in combination impact the plume statistics. Recall that before the screens were removed ARN2 had a quasi-turbulent peak turbulence intensity but a large (hence laminar-like) shape factor. After the screens were removed SMC000 had a quasi-laminar peak turbulence intensity, but a midsize, indeterminate shape factor of 2.20. From this we might expect that the two had different initial conditions, although neither was classically laminar or fully turbulent.

Figure 35 presents the centerline statistics for the mean and turbulent axial velocities of these two nozzles/test entries. Also shown is the Consensus dataset for this setpoint along with the uncertainty. Admittedly, some of the uncertainty was estimated based upon these two cases, but the uncertainty bars are in line with that of other setpoints and should be considered in that light. What does seem substantiated is that the differences in initial condition of the plumes have relatively 
small effect on these statistics, probably within measurement error at this point. Similar checks at higher Mach number show similar, or smaller, differences caused by the changes in rig and nozzle.
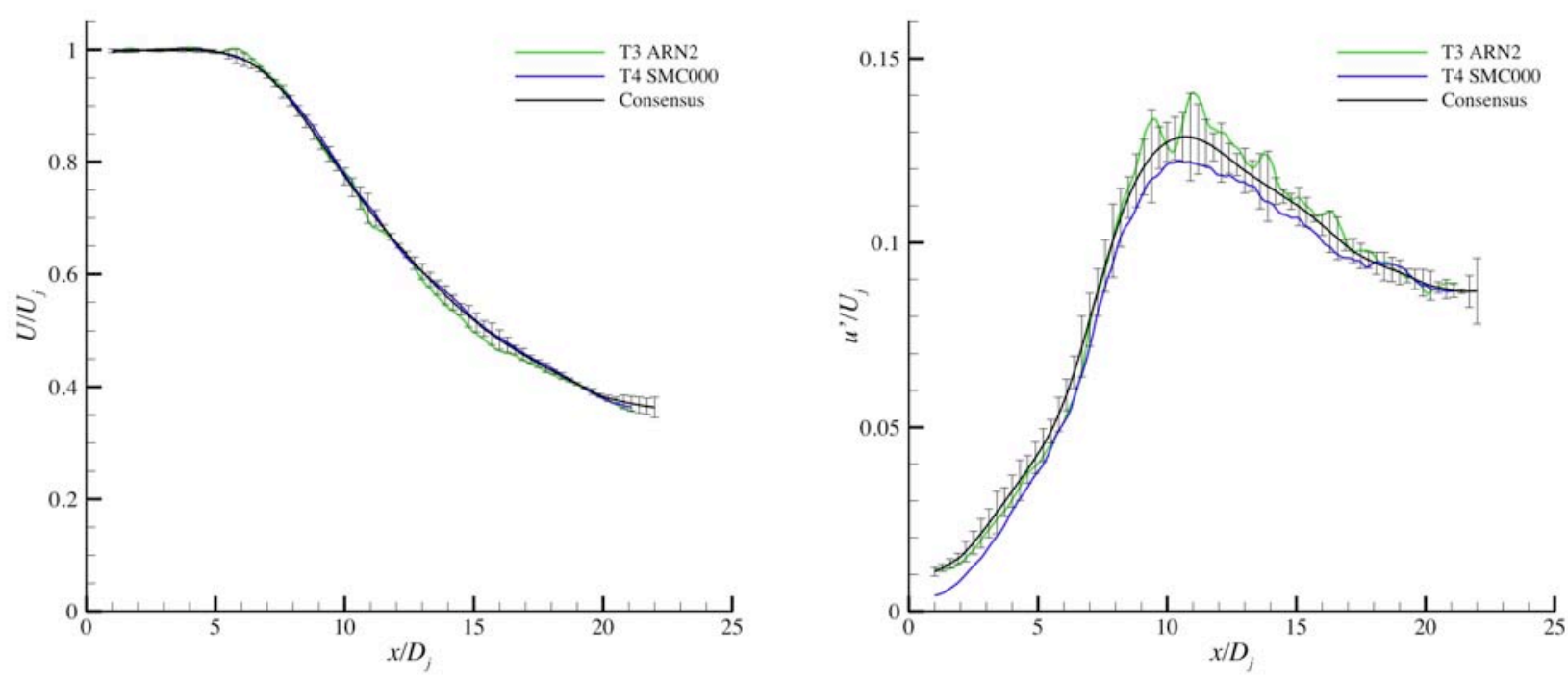

Figure 35 Comparison of centerline mean (left) and turbulent (right) axial velocities for different nozzle contraction types before and after rig screen changes and one year apart.

\section{Comparisons of Jet Scaling Parameters}

At this point, it is prudent to check the conformity of the data with certain expected scaling laws using appropriate dimensional parameters. In particular, the length of the potential core and the jet spread rate are key parameters which have been measured previously and are of technological importance. First, the axial scaling, summarized by the potential core length is examined using centerline plots. Success in this scaling is determined by considering the collapse of centerline plots of mean and turbulent velocities. Second, a scaling for the radial coordinate is sought using shear layer parameters, making the very simple assumption that the jet grows like a two-dimensional shear layer. The success of this scaling is demonstrated by radial profile plots of mean and turbulence velocity in shear layer coordinates. Finally, the jet is rescaled using both of these scalings together. A nice surprise occurs in that when the two scalings are combined all jet flows collapse to essentially one field.

\section{Potential Core Length}

The work of Witze [16] for predicting potential core lengths of jets is the first standard for comparison. In this paper a universal fit for subsonic mean centerline velocity is given by

$$
\frac{U}{U_{j}}=1-e^{\alpha /\left(1-x / X_{W}\right)} ; \quad \frac{X_{W}}{D_{j}}=\frac{4.375\left(\rho_{j} / \rho_{\infty}\right)^{0.28}}{1-0.16 M_{j}}
$$

The parameter $\alpha$ is 1.43 in accordance with Witze, and $X_{W}$ corresponds to a potential core length. Experimentally, the mean velocity noticeably falls off at roughly $x / X_{W}=1.3$. Normalizing by this potential core length should collapse all the centerline mean profiles, and if jet scaling is universal the peak turbulence intensity should also occur at a common $u^{\prime} / U_{j}$ for all jet flows.

The centerline profiles of the Consensus datasets are plotted together on coordinates normalized by the potential core length $X_{W}$ in Figure 36. While there is a substantial collapse, the agreement could be better. Corresponding to the variance in mean profiles, the locations of the peak axial turbulence on the centerline also disperse, the variance correlating with that of the mean profiles. While an improved measure for the potential core length could be found by rigorous curve fitting, an attempt was made to obtain better collapse by choosing potential core lengths that cause the turbulence to peak at the same location for all jets. This new potential core length, $X_{c}$, was used to renormalize the profiles as shown in Figure 37 . With this choice of potential core length the centerline mean profiles collapse on one another much better. The centerline axial turbulence profiles align by design, but the peak amplitudes differ. No further effort has been made to produce an improved correlation formula for potential core length at this time. 

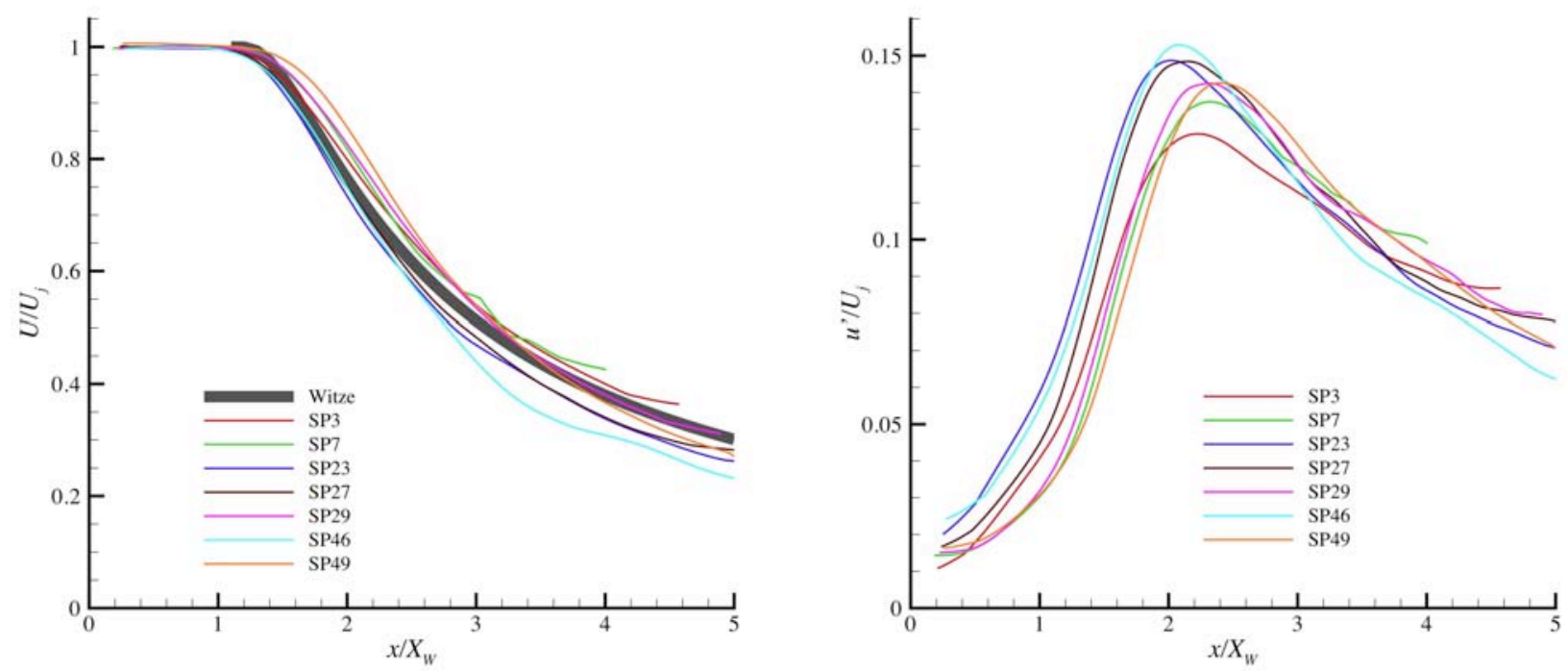

Figure 36 Centerline profiles of mean (right) and turbulent (left) axial velocity for all consensus datasets. Axial coordinate normalized by potential core length of Witze[16], $X_{W}$.
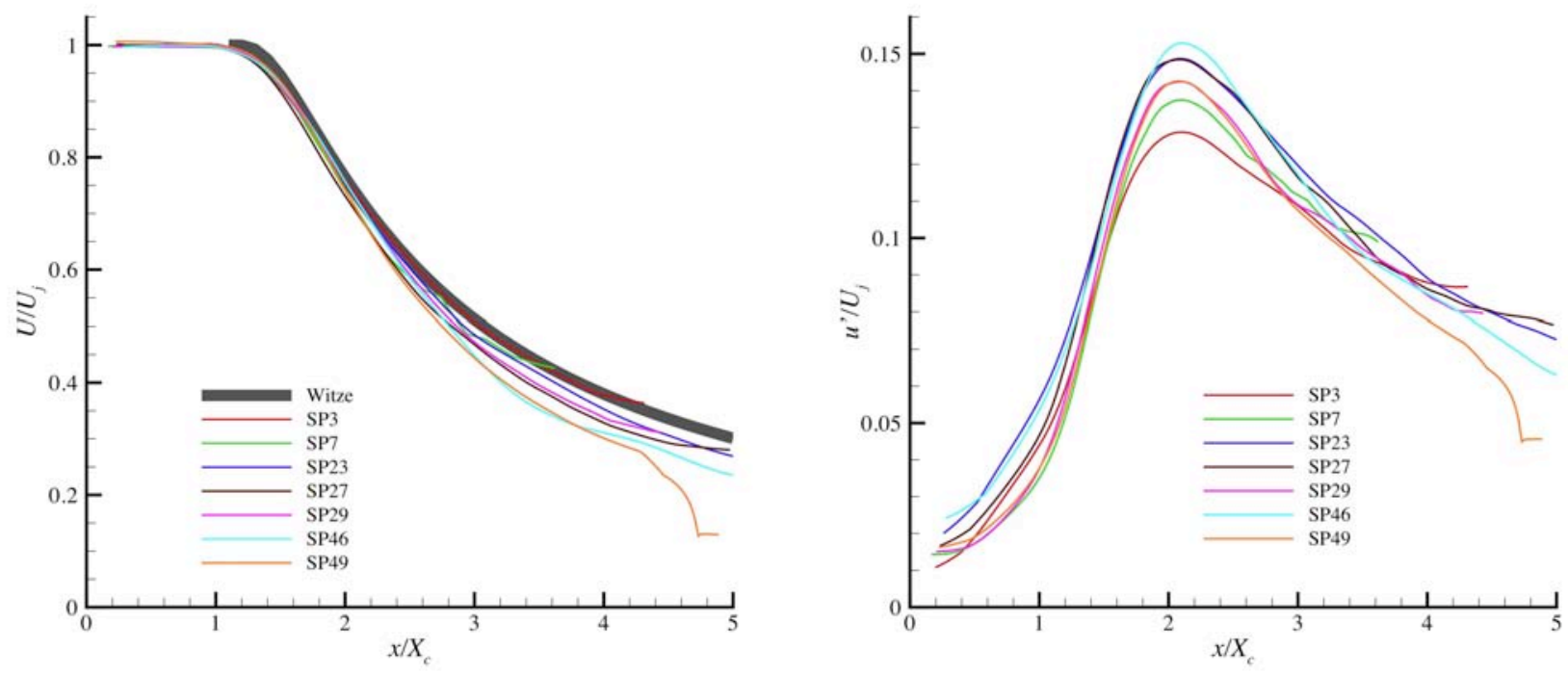

Figure 37 Centerline profiles of mean (right) and turbulent (left) axial velocity for all consensus datasets. Axial coordinate normalized by experimentally determined potential core length, $X_{c}$ 


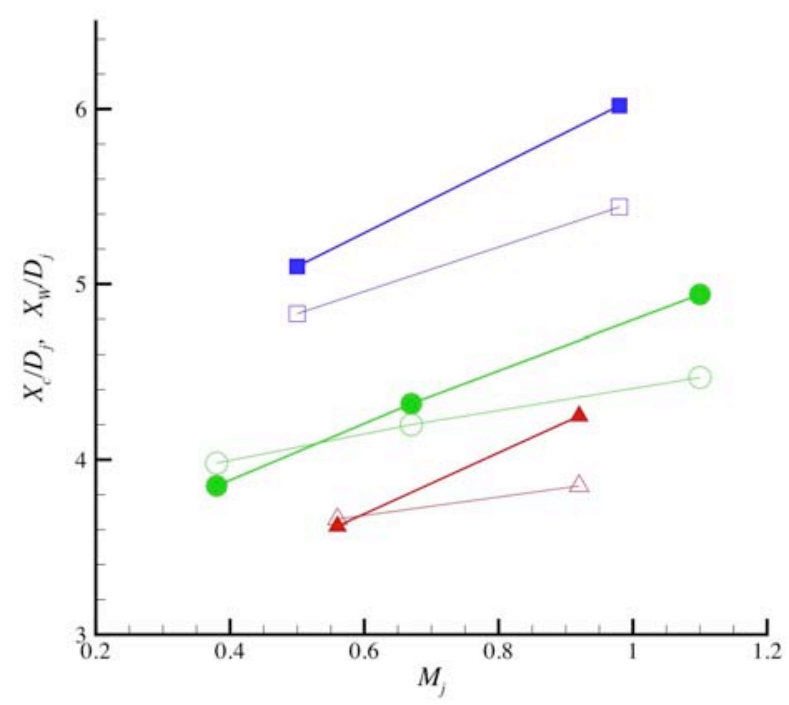

Figure 38 Potential core lengths as a function of Mach number for jets of different static temperature ratios: $\square$, unheated; $0,1.76 ; \triangle, 2.7$. Open symbols are $X_{W} / D_{j}$, solid symbols are $X_{c} / D_{j}$.

\section{Peak Turbulence Amplitude}

Aside from changes in potential core length, temperature and compressibility have only a small impact on subsonic jet turbulence amplitudes. A small trend exists that as potential lengths decreases, the centerline peak turbulence increases. This is illustrated in Figure 39 which shows the correlation between peak turbulence on the jet centerline and the potential core length for the jet flows in the Consensus dataset. The one outlier is the $M a=0.5$ unheated dataset. Also shown in the figure is the peak turbulence intensity over the entire jet plume for each jet, which correlates with the potential core length in a similar fashion.

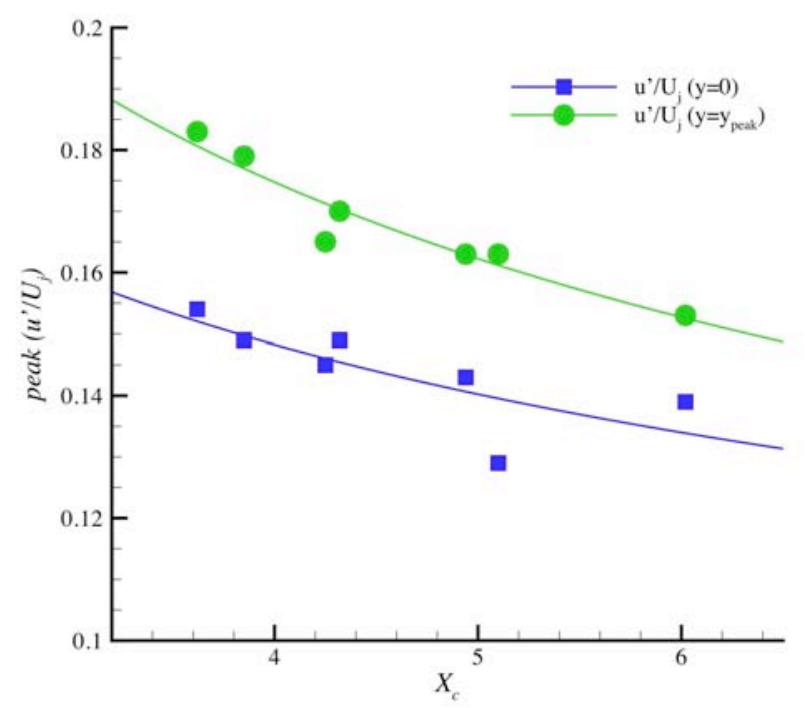

Figure 39 Peak axial turbulent velocity on centerline plotted against potential core length.

\section{Jet Spread}

While the potential core length is an important normalizing factor in jets, it is not the only difference caused by temperature and compressibility. The radial spread of the jet and the exponential decay region are also influenced in subtle ways that can be seen in this carefully cleaned data. In Figure 40 contour plots of the seven jet flow fields are overlaid, normalizing the axial coordinate by the experimentally obtained potential core length $X_{c}$. Examination of the plots reveals that, although the potential core lengths of all the datasets are the same by virtue of the normalization with $X_{c}$, the contours group by static temperature ratio or density. (Note that the line colors in the contour plots have been chosen to group jets with the same temperature ratio.) The unheated jets have a much greater spread rate than the heated jets and decay at a lower rate 
downstream. The grouping by static temperature ratio is a clear indication that it is density which drives these aspects of the flow, not the Mach number or compressibility: e.g. setpoints 23, 27, and 29 have aerodynamic Mach numbers $M_{j}=0.38,0.9$, 1.0 while maintaining the same static temperature ratio. The peak turbulence levels and their location are similarly affected by temperature, not Mach number. In Figure 40 the contour lines for a given turbulence level occur farther downstream and closer to the centerline with increased temperature.
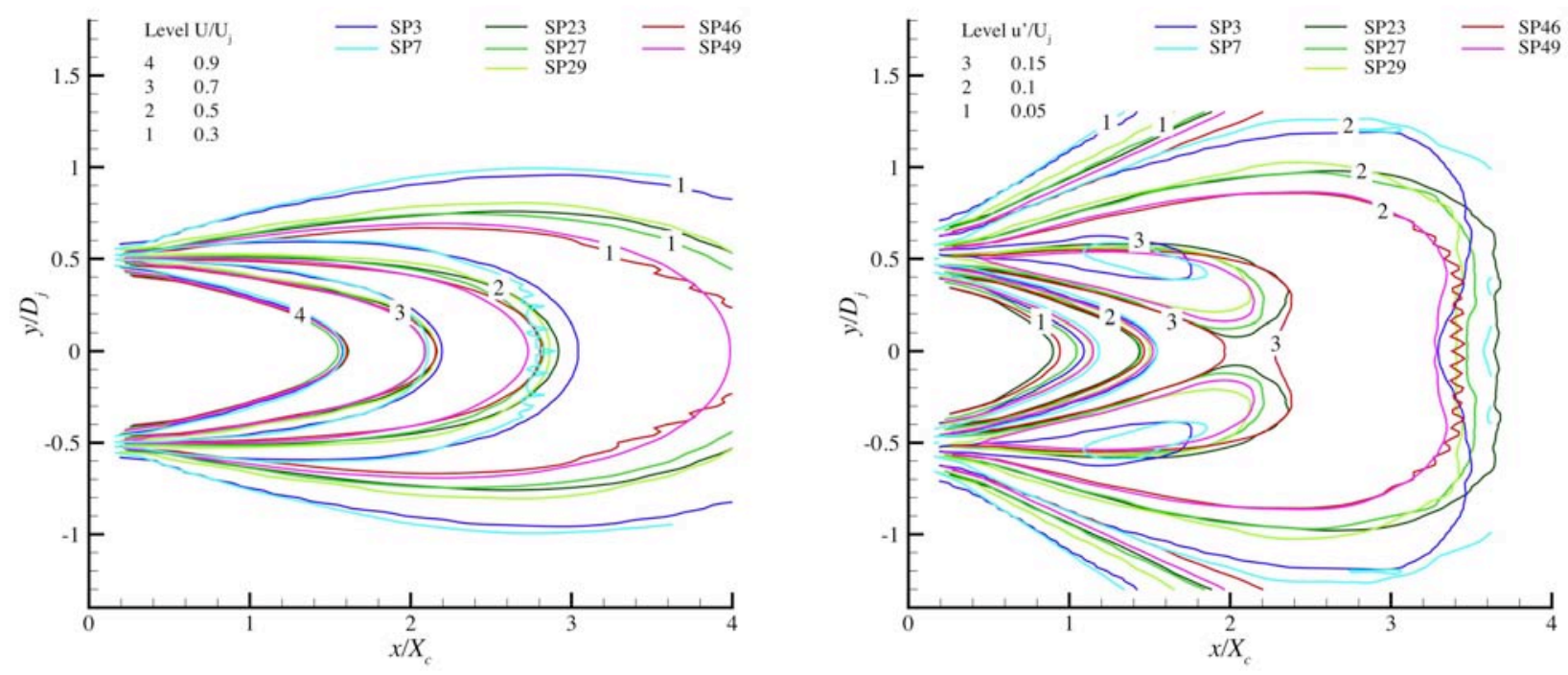

Figure 40 Mean and turbulent axial velocity contours for the seven consensus datasets, plotted with axial coordinate normalized by experimentally determined potential core length.

Perhaps a more direct way of seeing the impact of temperature is to look at radial profiles at given fractions of the potential core length. From these plots in Figure 41 it becomes apparent how the peak location of the turbulence (right half of each plot) tracks with the max shear of the mean velocity profiles (left half), causing the turbulence to peak in the cold jets further away from the centerline than in the hot jets. 

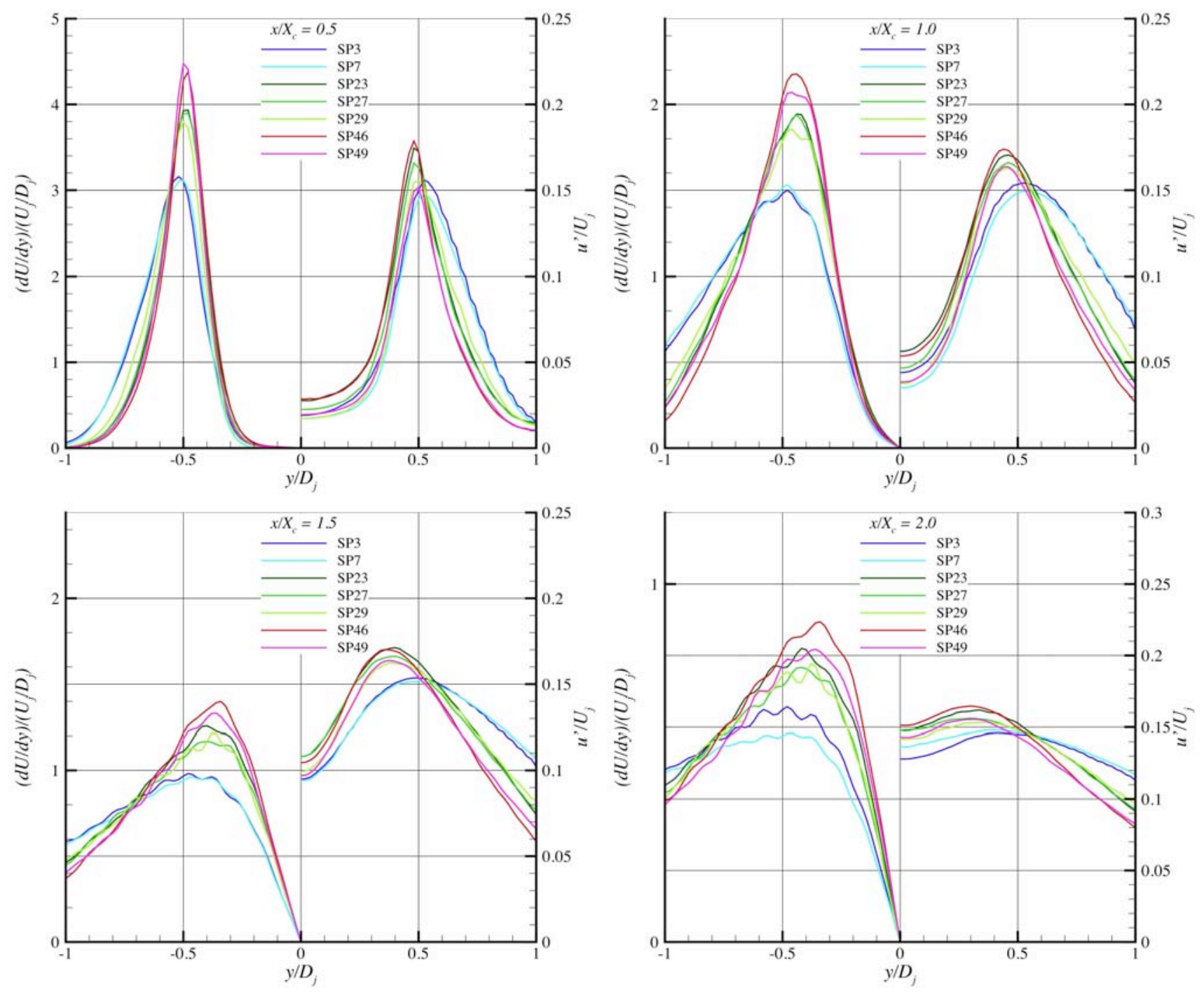

Figure 41 Radial profiles of consensus data for all setpoints at axial stations relative to potential core length $X_{c}$. Profiles plotted at $\boldsymbol{x} / X_{c}=0.5,1.0,1.5,2.0$.

The classic shear layer parameters of half-velocity point $r_{0.5}$ and shear layer thickness $\delta_{\omega}$ were calculated for each profile and are presented in Figure 42. The shear layer parameters were compared with those results published in Lau [10]. It appears that the Consensus data is a bit more self-consistent, but the general trends on change with Mach number and temperature ratio agree between the two datasets. As noted above, neither the half-velocity radius nor the shear layer thickness is a strong function of $M_{j}$, but only of temperature. These statistics were obtained for the streamwise location $x=X_{c}$, which is probably as far downstream as one can expect the jet shear layer to be approximately two-dimensional. Converting these parameters to growth rates, e.g. dividing the parameters by the axial distance, assuming this rate to be constant, one obtains the divergence angle of the half-velocity point $\psi=\left(r_{0.5}-D_{j} / 2\right) / x$ and the growth in the shear layer thickness $\delta_{\omega} x$. These are given in Figure 43 , again as a function of both temperature ratio and Mach number. From these plots it is seen that the jet spread $\psi$ varies considerably with temperature, but not Mach number. The spread rate $\delta_{\omega} x$, on the other hand, has some dependence on Mach number, but not temperature ratio. When plotted against Mach number $M_{j}$ the spread rate measured in the Consensus PIV datasets fall along a single line, relatively independent of temperature ratio. The data of Lau has similar trends, but has a 10\% greater spread rate than the Consensus data, and the data at the highest temperature $\left(T_{j} / T_{\infty}=2.32\right)$ does not fall in with the data of the two other temperature ratios. 

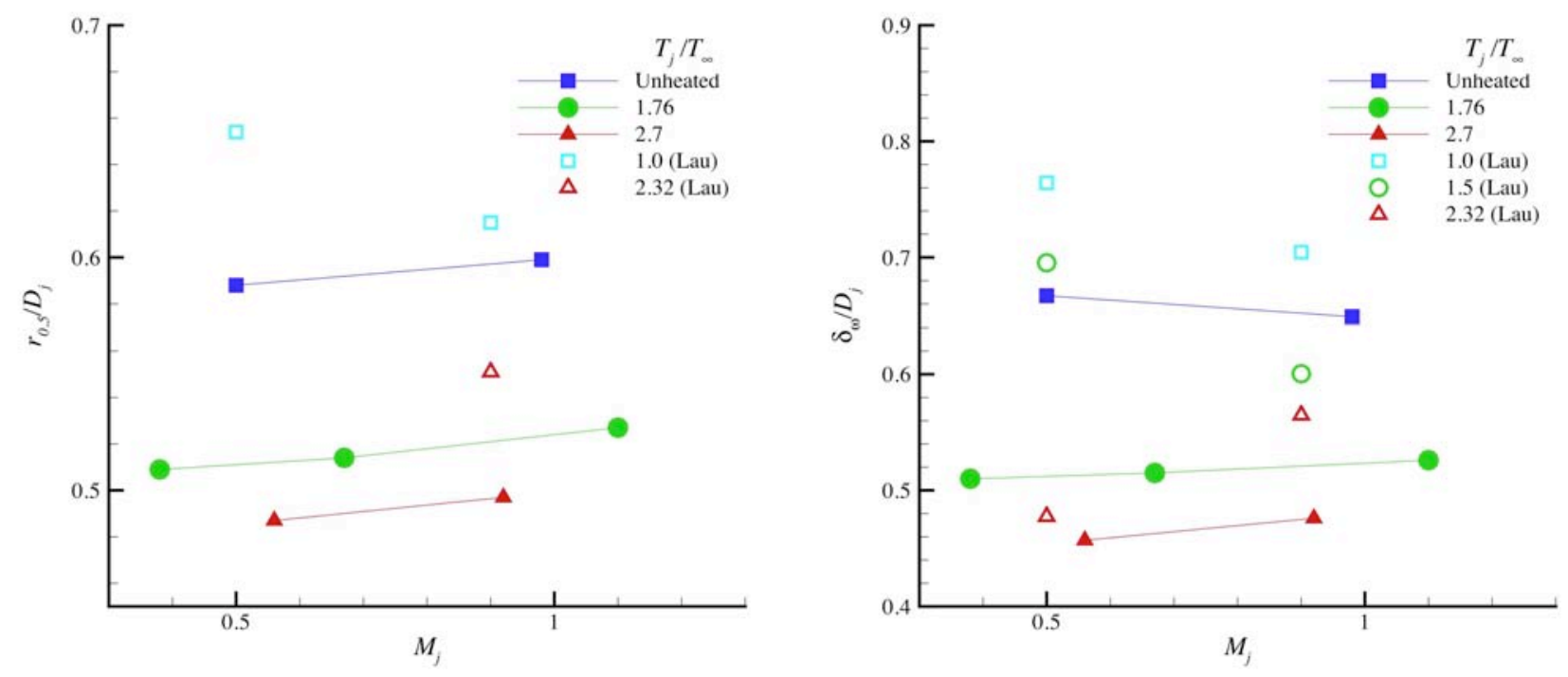

Figure 42 Shear layer parameters $r_{0.5}$ (jet half-width) and $\delta_{\omega}$ (shear layer thickness) as reported in Lau [10] and in Consensus datasets. Plots show dependence on static temperature ratio $T_{j} / T_{\infty}$ and on $M_{j}$ for $x=X_{c}$.
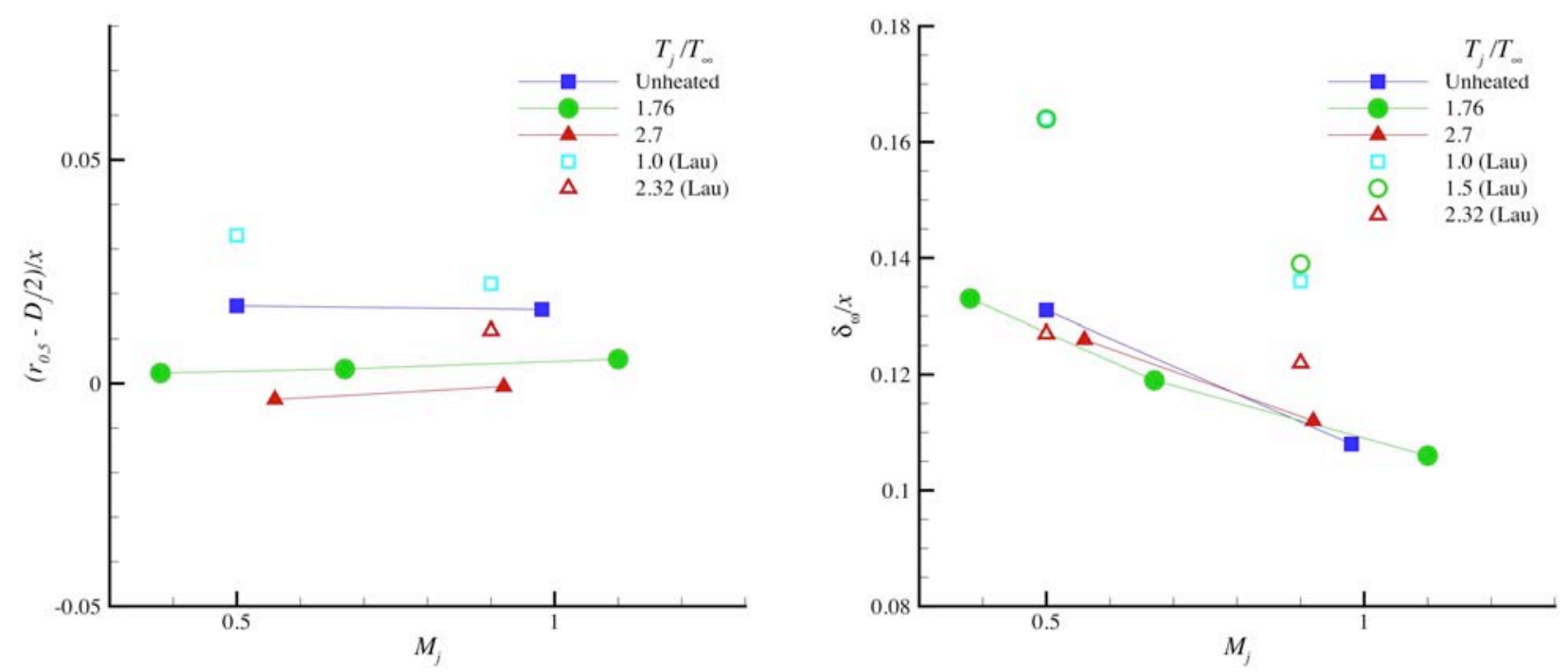

Figure 43 Shear layer growth parameters $\psi$ (jet half-width) and $\delta_{\omega} \boldsymbol{x}$ (shear layer thickness) with downstream distance as reported in Lau [10] and in Consensus datasets. Plots show dependence on static temperature ratio $T_{j} / T_{\infty}$ and on $M_{j}$.

\section{Combined Rescaling of Jet}

Having determined appropriate streamwise scaling from consideration of potential core length, and the shear layer scaling in terms of half-width and shear layer thickness, the contour plots of mean and turbulence velocity from all seven Consensus datasets are rescaled and overlaid as shown in Figure 44. The choice of streamwise coordinate is obvious, but the radial coordinate is not as straightforward. Using the standard shear layer coordinate $\eta$ is inappropriate because it does not preserve the jet axis. The cross-stream coordinate $y / Y_{c}$ used in the figure is essentially a simple radial stretch which maps the halfvelocity radius to the lipline of the jet. No consideration of the shear layer thickness is required-the shear layer thickness scales relatively well without it. This self-consistency shown in Figure 44 provides strong support both of traditional turbulence theory that gives rise to the normalization of shear layers, and of the Consensus datasets. The main area of disagreement is in the peak levels of the unheated jet, which are significantly lower than the other jets. The cause of this difference is not apparent at this time. 

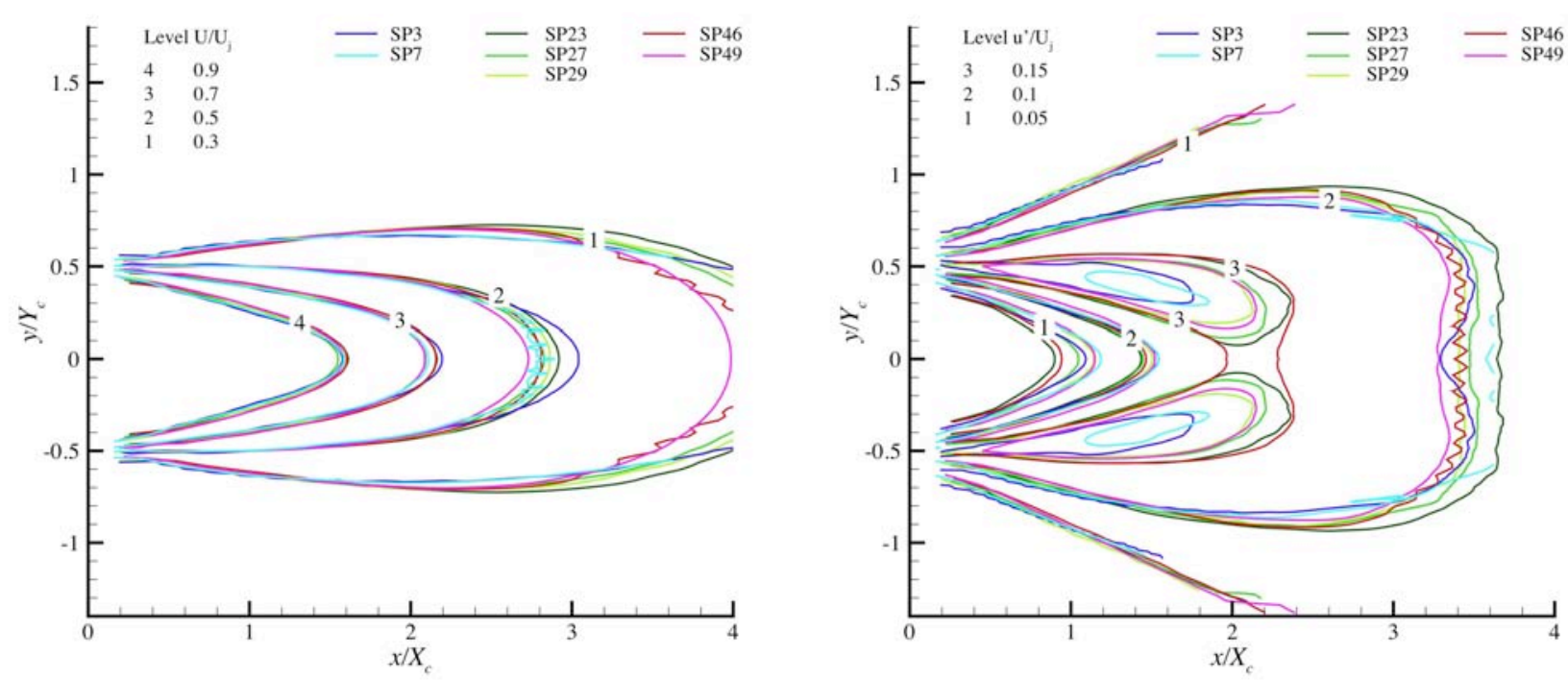

Figure 44 Mean and turbulent axial velocity contours for the seven consensus datasets, plotted with axial coordinate normalized by experimentally determined potential core length and transverse coordinate normalized to unify jet spread (half-velocity point).

\section{Summary}

A large body of Particle Image Velocimetry (PIV) data has been acquired over eight years of testing with different installations of optics and different generations of processing. Several flow cases covering a range of shock-free jets with different temperatures were repeatedly measured. The number of different test configurations and setups has produced opportunities to remove many bias errors associated with PIV configuration and the shear number of measurements reduces the random errors. Careful operation, including proper seeding techniques and close monitoring of flow conditions has also contributed to the high quality of the data.

This report details the experimental facility and the nozzle model hardware, including studies of the initial shear layer characteristics. It further covers all details of the PIV equipment, operations, and analysis during each test entry. It includes an extensive literature survey comparing turbulence data from many sources using different anemometry techniques. A subset of these data were chosen and justified for comparisons with the PIV datasets for cases of low subsonic cold, high subsonic cold, and high subsonic hot jets. This comparison provided an estimate of the bias error in the PIV data, e.g. the error that all the PIV data shared. It was found that the PIV turbulence intensity measurements fell within the $7 \%$ spread of the literature sources for the low subsonic cold jet with no appreciable bias, and within the $12 \%$ spread of the high subsonic cold jet with just a small bias to higher amplitudes if one does not include the LDV data sources from the literature. Very little basic turbulence data exists for high-speed jets and even less for high-speed hot jets. When the PIV data was overlaid on this data no appreciable bias was found.

A process of weighing, averaging, and smoothing the data from all the test entries was documented and used to obtain a 'Consensus' dataset for each flow case. The main objective of this Consensus dataset was to provide data for CFD validation and for studying subtle trends in the jet scaling, along with estimates of the uncertainty in the data. This dataset is intended to embody the best judgment of the experimentalist, freeing the end user from interpreting data irregularities. The raw data from all test entries were plotted along with the final Consensus dataset to establish random error estimates for the Consensus datasets.

Finally, the Consensus dataset was analyzed and standard characteristic measures of jets were compared with values from the literature. Potential core lengths, jet half-widths, peak turbulence levels, and shear layer growth rates were computed and compared with previous studies made using laser Doppler velocimetry. When the shear layer data for all jet flow cases were normalized using these appropriate parameters, the data from all flow cases collapsed in a satisfying manner.

\section{Acknowledgements}

The authors gratefully acknowledge the hard work and long hours put in by the technicians and engineers at the AeroAcoustics Propulsion Laboratory and by the research technicians who helped in setup and operation of the PIV systems. The data contained within this report were acquired primarily during the Quiet Aircraft Technology Program at NASA, and 
support of management for this fundamental effort is commended. Finally, the review of this manuscript by Dennis Yoder and Abbas Khavaran was very helpful in assuring accuracy and accessibility to the reader.

\section{References}

1 Laurence, J.C., Intensity, Scale, and Spectra of Turbulence In Mixing Regions of Free Subsonic Jet, NACA Report 1292 (1956).

2 Davies, P.O.A.L., Fisher \& M.J. \& Barratt, M.J., "The characteristics of the turbulence in the mixing region of a round jet," J. Fluid Mech. 15, 337 (1963).

3 Fisher, M. J. \& Davies, P. O. A. L. “Correlation measurements in a non-frozen pattern of turbulence,” J. Fluid Mech. 18, 97 (1964).

4 Bradshaw, P., Ferriss, D.H. \& Johnson, R.F., "Turbulence in the noise-producing region of a circular jet," J. Fluid Mech. 19, 591 (1964).

5 Ko, N.W.M. \& Davies, P.O.A.L., "The near field within the potential cone of subsonic cold jets," J. Fluid Mech. 60, 49 (1971).

6 Crow, S.C., \& Champagne, F.H., "Orderly structure in jet turbulence," J. Fluid Mech. 48, 547 (1971).

7 Raman, G., Zaman, K.B.M.Q., \& Rice, E.J., "Initial turbulence effect on jet evolution with and without tonal excitation," Phys Fluids A 11240 (1978).

8 Lau, J.C., Morris, P.J. \& Fisher, M. J., "Measurements in subsonic and supersonic free jets using a laser velocimeter," J. Fluid Mech. 93, 1. (1979).

9 Lau, J. C., "Laser velocimeter correlation measurements in subsonic and supersonic jets," J Sound Vibration 70, 85 (1980).

$10 \mathrm{Lau}, \mathrm{J}$. C., "Effects of exit Mach number and temperature on mean-flow and turbulence characteristics in round jets," J. Fluid Mech. 105, 193 (1981).

11 Ahuja, K.K., Lepicovsky, J., Tam, C.K.W., Morris, P.J., \& Burrin, R.H. “Tone-excited jet: Theory and experiments,” NASA CR3538 (1982).

12 Zaman, K.B.M.Q., Wang, F.Y. \& Georgiadis, N.J., "Noise, turbulence, and thrust of subsonic freejets from lobed nozzles,” AIAA J., 41, 398 (2003).

13 Kerherve, F., Jordan, P., Gervais, Y., Valie`re, J.-C., \& Braud, P., “Two-point laser Doppler velocimetry measurements in a Mach 1.2 cold supersonic jet for statistical aeroacoustic source model," Expt Fluids 37, 419 (2004). (2009).

14 Morris, P.J. \& Zaman, K.B.M.Q., "Velocity measurements in jets with application to noise source modeling," AIAA Paper 2009-17

15 Pokora, C.D. \& McGuirk, J.J., "Spatio-Temporal Turbulence Correlations using High-Speed PIV in an Axisymmetric Jet," AIAA Paper 2008-3028, (2008).

16 Witze, P.O., "Centerline velocity decay of compressible free jets," AIAA J 12, 417 (1974).

17 Viswanathan. K. "Jet aeroacoustic testing: issues and implications," AIAA J 41, 1674 (2003).

18 Ahuja. K.K., "Designing clean jet-noise facilities and making accurate jet-noise measurements," Intl J. Aeroacoustics 2,314 (2003).

19 Brown, C.A., \& Bridges, J., "Small Hot Jet Acoustic Rig Validation,” NASA TM-2006/214234 (2006).

20 Plumblee, H.E., Burrin, R.H., Lau, J.C., Morfey, C.L. Morris, P.J., Smith, D.M., Tanna, H.K., Tester, B.J., Whiffen, M.C., “The generation and radiation of supersonic jet noise, part III, Turbulent mixing noise data," AFAPL-TR-76-45, 1976.

21 Bridges, J., "Effect of Heat on Space-Time Correlations in Jets," AIAA Paper 2006-2534 (2006).

22 Bridges, J. \& Wernet, M.P., "Effect of Temperature on Jet Velocity Spectra," AIAA Paper 2007-3628, (2007).

23 Opalski, A.B., Wernet, M.P., \& Bridges, J., "Chevron nozzle performance characterization using stereoscopic DPIV," AIAA Paper 2005-0444, (2005).

24 Soloff, S.M., Adrian, R.J., \& Liu, Z-C, "Distortion Compensation for Generalized Stereoscopic Particle Image Velocimetry," Measurement Science and Technology 8, 1441, (1997).

25 Wernet, M.P., "Fuzzy Logic Enhanced Digital PIV Processing Software,” NASA TM—1999/209274, (1999).

26 Wernet, M. P., Skoch, G. J., \& Wernet, J. H., "Demonstration of a Stabilized Alumina/Ethanol Colloidal Dispersion Technique for Seeding High Temperature Air Flows," 16th International Congress on Instrumentation for Aerospace Simulation Facilities (ICIASF), (1995). 